\title{
CONTINUOUS AND DISCONTINUOUS GALERKIN TIME STEPPING METHODS FOR NONLINEAR INITIAL VALUE PROBLEMS WITH APPLICATION TO FINITE TIME BLOW-UP
}

\author{
BÄRBEL HOLM AND THOMAS P. WIHLER
}

\begin{abstract}
We consider continuous and discontinuous Galerkin time stepping methods of arbitrary order as applied to nonlinear initial value problems in real Hilbert spaces. Our only assumption is that the nonlinearities are continuous; in particular, we include the case of unbounded nonlinear operators. Specifically, we develop new techniques to prove general Peano-type existence results for discrete solutions. In particular, our results show that the existence of solutions is independent of the local approximation order, and only requires the local time steps to be sufficiently small (independent of the polynomial degree). The uniqueness of (local) solutions is addressed as well. In addition, our theory is applied to finite time blow-up problems with nonlinearities of algebraic growth. For such problems we develop a time step selection algorithm for the purpose of numerically computing the blow-up time, and provide a convergence result.
\end{abstract}

\section{INTRODUCTION}

In this paper we focus on continuous Galerkin (cG) as well as on discontinuous Galerkin (dG) time stepping discretizations (of any order) as applied to abstract initial value problems of the form

$$
u^{\prime}(t)=\mathcal{F}(t, u(t)), \quad t \in(0, T), \quad u(0)=u_{0} .
$$

Here, $u:(0, T) \rightarrow H$, for some $T>0$, is an unknown solution, with values in a real Hilbert space $H$ (with inner product denoted by $(\cdot, \cdot)_{H}$ and induced norm $\|\cdot\|_{H}$ ). The initial value $u_{0} \in H$ prescribes the solution $u$ at the start, $t=0$, and $\mathcal{F}$ : $[0, T] \times H \rightarrow H$ is a possibly nonlinear, continuous operator. We emphasize that we include, for instance, the case of $\mathcal{F}$ being (continuous and nonlinear and) unbounded in the sense that

$$
\frac{\|\mathcal{F}(t, x)\|_{H}}{\|x\|_{H}} \rightarrow \infty \text { as }\|x\|_{H} \rightarrow \infty, \quad 0 \leq t \leq T .
$$

In the sequel, we will usually omit to explicitly write the dependence on the first argument $t$.

For $H=\mathbb{R}^{N}$ and continuous nonlinearities $\mathcal{F}$, the well-known Peano Theorem (see, e.g., 17]) guarantees the existence of $C^{1}$-solutions $u$ of (1.1) within some

Department for Computational Science and Technology, School of Computer Science and Communication, KTH Royal Institute of Technology, SE-100 44 Stockholm, SWEDEN

Mathematisches Institut, Universität Bern, Sidlerstr. 5, CH-3012 Bern, Switzerland

E-mail addresses: barbel@kth.se, wihler@math.unibe.ch.

2010 Mathematics Subject Classification. 65J08, 65L05, 65L60.

Key words and phrases. Initial value problems in Hilbert spaces, Galerkin time stepping schemes, high-order methods, blow-up singularities, existence and uniqueness of Galerkin solutions.

The authors acknowledge the support of the Swiss National Science Foundation (SNF), Grant No. 200021-162990. 
limited time range, $t \in\left(0, T_{\infty}\right)$, for some $T_{\infty}>0$. Generalizations to problems in Banach spaces are available as well; see, e.g., [9]. Notice that the existence interval for solutions may be arbitrarily small even for smooth $\mathcal{F}$ : For instance, solutions of (1.1) may become unbounded in finite time, i.e.,

$$
\|u(t)\|_{H}<\infty \text { for } 0<t<T_{\infty}, \quad \lim _{t \nearrow T_{\infty}}\|u(t)\|_{H}=\infty .
$$

This effect is commonly termed (finite-time) blow-up.

Galerkin Time Stepping. Galerkin-type time stepping methods for initial-value problems are based on weak formulations. For both the $\mathrm{cG}$ and the $\mathrm{dG}$ time stepping schemes, the test spaces consist of polynomials that are discontinuous at the time nodes. In this way, the discrete Galerkin formulations decouple into local problems on each time step, and the discretizations can therefore be understood as implicit one-step schemes. Galerkin time stepping methods have been analyzed for ordinary differential equations (ODEs), e.g., in [3, 5, 10,

A key feature of Galerkin time stepping methods is their great flexibility with respect to the size of the time steps and the local approximation orders, thereby naturally leading to an $h p$-version Galerkin framework. The $h p$-versions of the cG and $\mathrm{dG}$ time stepping schemes were introduced and analyzed in the works [12,13, 15, 19]. In particular, in the articles [12,19, which focus on ordinary initial value problems with uniform Lipschitz nonlinearities, the use of the contraction mapping theorem made it possible to prove existence and uniqueness results for discrete Galerkin solutions, which are independent of the local approximation orders. We emphasize that the $h p$-approach is well-known for its ability to approximate smooth solutions with possible local singularities at high algebraic or even exponential rates of convergence; see, e.g., 4, 13, 14, 18, for the numerical approximation of problems with start-up singularities.

Results. The goal of the current paper is to extend the existence results on $h p$-type Galerkin time stepping schemes for initial value problems with Lipschitz-type nonlinearities in [12,19] to problems with nonlinearities which are merely continuous. We emphasize that this generalization is substantial; indeed, it covers, for example, the case of unbounded nonlinearities as in (1.2). We will develop a new technique which is based on writing the weak Galerkin formulations in strong form along the lines of 1, 15. Subsequently, suitable fixed-point forms will be derived. In the context of the $\mathrm{cG}$ method, this is accomplished within an integral equation framework. For the dG scheme, matters are more sophisticated, and a careful investigation of the discrete time derivative operator, which involves a lifting operator from [15], is required on the local polynomial approximation space; this operator turns out to be an isomorphism on the underlying polynomial spaces (with a continuity constant of the inverse operator that is independent of the local polynomial degrees) and allows to transform the strong $\mathrm{dG}$ form into a fixed point equation. For both the $\mathrm{cG}$ and the $\mathrm{dG}$ schemes the application of Brower's fixed point theorem yields the existence of discrete solutions; see Theorem 1 In particular, as in the case of Lipschitz continuous nonlinearities [1,15, the existence results do not depend on the local polynomial degrees, and only require the local time steps to be sufficiently small. In this sense, our theory constitutes a discrete version of Peano's Theorem. Furthermore, employing a contraction argument along the lines of the approach presented in [2], we show that the local Galerkin formulations are uniquely solvable (within a certain range); cf. Theorem 2 .

In addition, we apply our general theory to initial value problems with nonlinearities of algebraic growth, i.e., $\mathcal{F}(t, u) \sim \alpha\|u\|_{H}^{\beta}$, with $\alpha>0, \beta>1$, and for a given range of $t$; in this case, the initial value problem (1.1) features a solution 
that blows up in a finite time $T_{\infty}$. We will show that a careful selection of locally varying time steps in the $\mathrm{cG}$ and $\mathrm{dG}$ time stepping schemes results in discrete solutions that blow up as well; in this context, we mention the paper [16] which illustrates the importance of variable step size selection. More precisely, following some ideas from [11, we derive an analysis which allows to choose the local time steps a posteriori as the time marching process is moving forward. We develop a time step selection algorithm which guarantees the existence and uniqueness of local solutions, and provides a numerical approximation of the exact blow-up time. Moreover, we prove a convergence result which shows that the blow-up time can be approximately arbitrarily well if the time steps are scaled sufficiently small.

The concepts and technical tools developed in our current work constitute an important stepping stone with regard to the numerical treatment of finite time blowup problems in the context of nonlinear parabolic partial differential equations.

Outline. Our article is organized as follows: Section 2 presents the cG and dG time stepping schemes. Furthermore, Section 3 centres on the development of existence proofs for discrete solutions. The question of uniqueness is addressed in Section 4. Moreover, the application of our results to algebraically growing nonlinearities causing finite time blow-ups will be worked out in Section 5 . Finally, the article closes with a few concluding remarks in Section 6.

Notation. Throughout the paper, Bochner spaces will be used: For an interval $I=(a, b)$ and a real Hilbert space $H$ as before, the space $C^{0}(\bar{I} ; H)$ consists of all functions $u: \bar{I} \rightarrow H$ that are continuous on $\bar{I}$ with values in $H$. Moreover, introducing, for $1 \leq p \leq \infty$, the norm

$$
\|u\|_{L^{p}(I ; H)}= \begin{cases}\left(\int_{I}\|u(t)\|_{H}^{p} \mathrm{~d} t\right)^{1 / p}, & 1 \leq p<\infty \\ {\operatorname{ess} \sup _{t \in I}\|u(t)\|_{H},}, \quad p=\infty\end{cases}
$$

we write $L^{p}(I ; H)$ to signify the space of measurable functions $u: I \rightarrow H$ so that the corresponding norm is bounded. We notice that $L^{2}(I ; H)$ is a Hilbert space with inner product and induced norm

$$
(u, v)_{L^{2}(I ; H)}=\int_{I}(u(t), v(t))_{H} \mathrm{~d} t, \quad \text { and } \quad\|u\|_{L^{2}(I ; H)}=\left(\int_{I}\|u(t)\|_{H}^{2} \mathrm{~d} t\right)^{1 / 2}
$$

respectively.

\section{Galerkin Time Discretizations}

In this section we present the $h p$-cG and $h p$-dG time stepping methods as applied to (1.1).

2.1. $h p$-cG Time Stepping. On an interval $I=[0, T], T>0$, consider time nodes $0=t_{0}<t_{1}<\cdots<t_{M-1}<t_{M}=T$ which introduce a time partition $\mathcal{M}=\left\{I_{m}\right\}_{m=1}^{M}$ of $I$ into $M$ open time intervals $I_{m}=\left(t_{m-1}, t_{m}\right), m=1, \ldots, M$. The (possibly varying) length $k_{m}=t_{m}-t_{m-1}$ of a time interval is called the $m^{\text {th }}$ time step. Furthermore, to each interval we associate a polynomial degree $r_{m} \geq 0$ which takes the role of a local approximation order. Moreover, given a (real) Hilbert space $X \subset H$, an integer $r \in \mathbb{N}_{0}$, and an interval $J \subset \mathbb{R}$, the set

$$
\mathcal{P}^{r}(J ; X)=\left\{p \in C^{0}(\bar{J} ; X): p(t)=\sum_{i=0}^{r} x_{i} t^{i}, x_{i} \in X\right\}
$$

signifies the space of all polynomials of degree at most $r$ on $J$ with values in $X$. 
In practical computations, the Hilbert space $H$, on which (1.1) is based, will typically be replaced by a finite-dimensional subspace $H_{m} \subset H, \operatorname{dim}\left(H_{m}\right)<\infty$, on each interval $I_{m}, 1 \leq m \leq M$. The $H$-orthogonal projection from $H$ to $H_{m}$ is defined by

$$
\pi_{m}: H \rightarrow H_{m}, \quad\left(x-\pi_{m} x, y\right)_{H}=0 \quad \forall y \in H_{m} .
$$

With these definitions, the (fully discrete) $h p$-cG time marching scheme is iteratively given as follows: For given initial value $U_{m-1}:=\left.\lim _{t} t_{t_{m-1}} U\right|_{I_{m-1}}(t) \in H$ (with $U_{0}:=u_{0}$, where $u_{0} \in H$ is the initial value from (1.1)), we find $\left.U\right|_{I_{m}} \in$ $\mathcal{P}^{r_{m}+1}\left(I_{m} ; H_{m}\right)$ through the weak formulation

$$
\begin{aligned}
\int_{I_{m}}\left(U^{\prime}, V\right)_{H} \mathrm{~d} t & =\int_{I_{m}}(\mathcal{F}(U), V)_{H} \mathrm{~d} t \quad \forall V \in \mathcal{P}^{r_{m}}\left(I_{m} ; H_{m}\right), \\
U\left(t_{m-1}\right) & =\pi_{m} U_{m-1},
\end{aligned}
$$

for any $1 \leq m \leq M$. Notice that, in order to enforce the initial condition on each individual time step, the local trial space has one degree of freedom more than the local test space. Furthermore, if $H_{1}=H_{2}=\ldots=H_{M}$, we remark that the continuous Galerkin solution $U$ is globally continuous on $(0, T)$.

2.2. $h p$-dG Time Stepping. In order to define the discontinuous Galerkin scheme, some additional notation is required: We define the one-sided limits of a piecewise continuous function $U$ at each time node $t_{m}$ by

$$
U_{m}^{+}:=\lim _{s \searrow 0} U\left(t_{m}+s\right), \quad U_{m}^{-}:=\lim _{s \searrow 0} U\left(t_{m}-s\right) .
$$

Then, the discontinuity jump of $U$ at $t_{m}, 0 \leq m \leq M-1$, is defined by $\llbracket U \rrbracket_{m}:=$ $U_{m}^{+}-U_{m}^{-}$, where we let $U_{0}^{-}:=u_{0}$, with $u_{0}$ being the initial condition from (1.1). Then, the (fully discrete) $h p$-dG time stepping method for (1.1) reads: Find $\left.U\right|_{I_{m}} \in$ $\mathcal{P}^{r_{m}}\left(I_{m} ; H_{m}\right)$ such that

$$
\int_{I_{m}}\left(U^{\prime}, V\right)_{H} \mathrm{~d} t+\left(\llbracket U \rrbracket_{m-1}, V_{m-1}^{+}\right)_{H}=\int_{I_{m}}(\mathcal{F}(U), V)_{H} \mathrm{~d} t \quad \forall V \in \mathcal{P}^{r_{m}}\left(I_{m} ; H_{m}\right),
$$

for any $1 \leq m \leq M$. We emphasize that, in contrast to the continuous Galerkin formulation, the trial and test spaces are the same for the discontinuous Galerkin scheme. This is due to the fact that the initial values are weakly imposed (by means of an upwind flux) on each time interval.

\section{Existence of Discrete Solutions}

In this Section our goal is to show existence of solutions to the discrete local problems (2.2) and (2.3):

Theorem 1. Let $m \geq 1$. Then, if the local time step $k_{m}>0$ is chosen sufficiently small (independent of the local polynomial degree $r_{m}$ ), then the continuous Galerkin method (2.2) and the discontinuous Galerkin method (2.3) on $I_{m}$ both possess at least one solution $U_{\mathrm{cG}} \in \mathcal{P}^{r_{m}+1}\left(I_{m} ; H_{m}\right)$ and $U_{\mathrm{dG}} \in \mathcal{P}^{r_{m}}\left(I_{m} ; H_{m}\right)$, respectively.

Our general strategy of proof is to represent the Galerkin formulations in terms of strong equations, and then to derive suitable fixed-point formulations. Subsequently, the existence of discrete solutions will follow from the application of Brower's fixed point theorem. 
3.1. Existence of $\mathbf{c G}$ Solutions. We begin by rewriting (2.2) as finding $U \in$ $\mathcal{P}^{r_{m}+1}\left(I_{m} ; H_{m}\right)$ such that

$$
\begin{gathered}
\int_{I_{m}}\left(U^{\prime}-\Pi_{m}^{r_{m}} \mathcal{F}(U), V\right)_{H} \mathrm{~d} t=0 \quad \forall V \in \mathcal{P}^{r_{m}}\left(I_{m} ; H_{m}\right), \\
U\left(t_{m-1}\right)=\pi_{m} U_{m-1} .
\end{gathered}
$$

Here, $\Pi_{m}^{r_{m}}: L^{2}\left(I_{m} ; H\right) \rightarrow \mathcal{P}^{r_{m}}\left(I_{m} ; H_{m}\right)$ denotes the $L^{2}$-projection onto the space $\mathcal{P}^{r_{m}}\left(I_{m} ; H_{m}\right)$, which is uniquely defined by

$$
u \mapsto \Pi_{m}^{r_{m}} u: \quad \int_{I_{m}}\left(u-\Pi_{m}^{r_{m}} u, V\right)_{H} \mathrm{~d} t=0 \quad \forall V \in \mathcal{P}^{r_{m}}\left(I_{m} ; H_{m}\right) .
$$

Thence, noticing that $U^{\prime}-\Pi_{m}^{r_{m}} \mathcal{F}(U) \in \mathcal{P}^{r_{m}}\left(I_{m} ; H_{m}\right)$, we obtain the strong form

$$
\begin{aligned}
U^{\prime}-\Pi_{m}^{r_{m}} \mathcal{F}(U) & =0 \quad \text { on } I_{m}, \\
U\left(t_{m-1}\right) & =\pi_{m} U_{m-1} .
\end{aligned}
$$

Integration results in

$$
U(t)=\pi_{m} U_{m-1}+\int_{t_{m-1}}^{t} \Pi_{m}^{r_{m}} \mathcal{F}(U) \mathrm{d} \tau, \quad t \in I_{m} .
$$

We see that the operator

$$
\mathrm{T}_{m}^{\mathrm{cG}}(U)(t):=\pi_{m} U_{m-1}+\int_{t_{m-1}}^{t} \Pi_{m}^{r_{m}} \mathcal{F}(U) \mathrm{d} \tau
$$

maps $\mathcal{P}^{r_{m}+1}\left(I_{m} ; H_{m}\right)$ into itself, and hence, the integral equation (3.2) is a fixed point formulation,

$$
\mathrm{T}_{m}^{\mathrm{cG}}(U)=U
$$

on $\mathcal{P}^{r_{m}+1}\left(I_{m} ; H_{m}\right)$. In particular, any solution of (3.2) will solve (2.2).

We are now ready to prove Theorem 1 for the continuous Galerkin method (2.2): For some $\kappa_{m}, \theta_{m}>0$ (with $t_{m-1}+\theta_{m} \leq T$ ) let us define the set

$$
Q_{m}=\left[t_{m-1}, t_{m-1}+\theta_{m}\right] \times B_{m}
$$

where

$$
B_{m}=\left\{y \in H_{m}:\left\|y-\pi_{m} U_{m-1}\right\|_{H} \leq \kappa_{m}\right\} .
$$

Since $\mathcal{F}$ is continuous, its maximum on the compact set $Q_{m}$,

$$
K_{m}:=\max _{(t, y) \in Q_{m}}\|\mathcal{F}(t, y)\|_{H},
$$

exists. We let

Then, we introduce

$$
0<k_{m} \leq \min \left(\theta_{m}, K_{m}^{-1} \kappa_{m}\right)
$$

$$
M_{m}^{\mathrm{cG}}:=\left\{Y \in \mathcal{P}^{r_{m}+1}\left(I_{m} ; H_{m}\right): Y(t) \in B_{m} \forall t \in \bar{I}_{m}\right\},
$$

where $I_{m}=\left(t_{m-1}, t_{m}\right)$, with $t_{m}=t_{m-1}+k_{m}$.

Let $U \in M_{m}^{\mathrm{cG}}$ be arbitrary, and $t^{\star} \in \bar{I}_{m}$ such that

$$
\left\|\mathrm{T}_{m}^{\mathrm{cG}}(U)\left(t^{\star}\right)-\pi_{m} U_{m-1}\right\|_{H}=\left\|\mathrm{T}_{m}^{\mathrm{cG}}(U)-\pi_{m} U_{m-1}\right\|_{L^{\infty}\left(I_{m} ; H\right)} .
$$

Then, using Bochner's Theorem as well as the Cauchy-Schwarz inequality, yields

$$
\begin{aligned}
\left\|\mathrm{T}_{m}^{\mathrm{cG}}(U)-\pi_{m} U_{m-1}\right\|_{L^{\infty}\left(I_{m} ; H\right)} & \leq\left\|\int_{t_{m-1}}^{t^{\star}} \Pi_{m}^{r_{m}} \mathcal{F}(U) \mathrm{d} \tau\right\|_{H} \leq \int_{I_{m}}\left\|\Pi_{m}^{r_{m}} \mathcal{F}(U)\right\|_{H} \mathrm{~d} \tau \\
& \leq k_{m}^{1 / 2}\left\|\Pi_{m}^{r_{m}} \mathcal{F}(U)\right\|_{L^{2}\left(I_{m} ; H\right)} .
\end{aligned}
$$


Taking into account the boundedness of the $L^{2}$-projection on $I_{m}$ (with constant 1 ) leads to

$$
\left\|\mathrm{T}_{m}^{\mathrm{cG}}(U)-\pi_{m} U_{m-1}\right\|_{L^{\infty}\left(I_{m} ; H\right)} \leq k_{m}^{1 / 2}\|\mathcal{F}(U)\|_{L^{2}\left(I_{m} ; H\right)} \leq k_{m}\|\mathcal{F}(U)\|_{L^{\infty}\left(I_{m} ; H\right)} .
$$

Therefore,

$$
\left\|\mathrm{T}_{m}^{\mathrm{cG}}(U)-\pi_{m} U_{m-1}\right\|_{L^{\infty}\left(I_{m} ; H\right)} \leq K_{m} k_{m} \leq \kappa_{m}
$$

Thus, we have $\mathrm{T}_{m}^{\mathrm{cG}}(U) \in M_{m}^{\mathrm{cG}}$, and more generally, it follows $\mathrm{T}_{m}^{\mathrm{cG}}\left(M_{m}^{\mathrm{cG}}\right) \subseteq M_{m}^{\mathrm{cG}}$. Finally, since $M_{m}^{\mathrm{cG}}$ is convex and compact, and $\mathrm{T}_{m}^{\mathrm{cG}}$ is continuous, Brower's fixed point theorem implies that there exists at least one solution of (3.4) in $M_{m}^{\mathrm{cG}}$, and thus of (2.2).

3.2. Existence of $\mathbf{d G}$ Solutions. The situation for the dG method is more involved. We will commence by looking at a discrete dG time operator appearing in the $\mathrm{dG}$ formulation.

3.2.1. Discrete dG Time Operator. Following [15, Section 4.1] we define the lifting operator, for $1 \leq m \leq M$,

$$
\mathrm{E}_{m}^{r_{m}}: X \rightarrow \mathcal{P}^{r_{m}}\left(I_{m} ; X\right)
$$

by

$$
\int_{I_{m}}\left(\mathrm{E}_{m}^{r_{m}}(z), V\right)_{X} \mathrm{~d} t=\left(z, V_{m-1}^{+}\right)_{X} \quad \forall V \in \mathcal{P}^{r_{m}}\left(I_{m} ; X\right), z \in X,
$$

on a real Hilbert space $X$, with inner product $(\cdot, \cdot)_{X}$, and norm $\|\cdot\|_{X}$.

In view of this definition with $X=H_{m}$, we have for the $\mathrm{dG}$ solution $U \in$ $\mathcal{P}^{r_{m}}\left(I_{m} ; H_{m}\right)$ from (2.3):

$$
\begin{aligned}
0 & =\int_{I_{m}}\left\{\left(U^{\prime}, V\right)_{H}-(\mathcal{F}(U), V)_{H}\right\} \mathrm{d} t+\left(\llbracket U \rrbracket_{m-1}, V_{m-1}^{+}\right)_{H} \\
& =\int_{I_{m}}\left\{\left(U^{\prime}, V\right)_{H}-\left(\Pi_{m}^{r_{m}} \mathcal{F}(U), V\right)_{H}\right\} \mathrm{d} t+\left(\pi_{m} \llbracket U \rrbracket_{m-1}, V_{m-1}^{+}\right)_{H} \\
& =\int_{I_{m}}\left\{\left(U^{\prime}+\mathrm{七}_{m}^{r_{m}}\left(\pi_{m} \llbracket U \rrbracket_{m-1}\right)-\Pi_{m}^{r_{m}} \mathcal{F}(U), V\right)_{H}\right\} \mathrm{d} t,
\end{aligned}
$$

for any $V \in \mathcal{P}^{r_{m}}\left(I_{m} ; H_{m}\right)$. Here, $\pi_{m}$ is the $H$-orthogonal projection from (2.1), and $\Pi_{m}^{r_{m}}$ is the $L^{2}$-projection from (3.1).

Then, since $U^{\prime}, \mathrm{E}_{m}^{r_{m}}\left(\pi_{m} \llbracket U \rrbracket_{m-1}\right), \Pi_{m}^{r_{m}} \mathcal{F}(U)$ all belong to $\mathcal{P}^{r_{m}}\left(I_{m} ; H_{m}\right)$, we arrive at the strong formulation

$$
U^{\prime}+\mathrm{E}_{m}^{r_{m}}\left(\pi_{m} \llbracket U \rrbracket_{m-1}\right)=\Pi_{m}^{r_{m}} \mathcal{F}(U)
$$

of (2.3). The term on the left-hand side of this equation is the $h p-\mathrm{dG}$ time discretization of the continuous derivative operator $u \mapsto u^{\prime}$. This motivates the definition of a discrete operator

given by

$$
\chi: \mathcal{P}^{r_{m}}\left(I_{m} ; H_{m}\right) \rightarrow \mathcal{P}^{r_{m}}\left(I_{m} ; H_{m}\right)
$$

$$
U \mapsto \chi(U)=U^{\prime}+\mathrm{E}_{m}^{r_{m}}\left(U_{m-1}^{+}\right) .
$$

For the proof of existence of solutions of (3.8) it is important to notice that the linear operator $\chi$ is invertible.

Proposition 1. Let $X$ be a real Hilbert space, and $1 \leq m \leq M$. Then, the operator $\chi$ from (3.9) 3.10 is an isomorphism on $\mathcal{P}^{r_{m}}\left(\bar{I}_{m} ; X\right)$. In addition, there exists a constant $C_{\chi}>0$ independent of the time step $k_{m}$ and the local approximation order $r_{m}$ such that, for any $p \in[1, \infty]$, there holds the bound

$$
\|U\|_{L^{\infty}\left(I_{m} ; X\right)} \leq C_{\chi} k_{m}^{1-1 / p}\|\chi(U)\|_{L^{p}\left(I_{m} ; X\right)},
$$

for any $U \in \mathcal{P}^{r_{m}}\left(I_{m} ; X\right)$. 
In order to establish this estimate, we require two auxiliary results which will be proved first.

Lemma 1. Let $1 \leq m \leq M$, and $X$ a real Hilbert space. Then, there holds

$$
\sup _{t \in I_{m}}\left\|z-\int_{t_{m-1}}^{t} E_{m}^{r_{m}}(z) \mathrm{d} \tau\right\|_{X}=\|z\|_{X}
$$

for any $z \in X$.

Proof. Let us first consider the lifting operator $\widehat{\mathrm{E}}^{r_{m}}: X \rightarrow \mathcal{P}^{r_{m}}(\widehat{I} ; X)$ on the unit interval $\widehat{I}=(-1,1)$, defined by

$$
\int_{-1}^{1}\left(\widehat{\mathrm{E}}^{r_{m}}(z), \widehat{V}\right)_{X} \mathrm{~d} \hat{t}=(z, \widehat{V}(-1))_{X} \quad \forall \widehat{V} \in \mathcal{P}^{r_{m}}(\widehat{I} ; X), z \in X .
$$

Referring to [15, Eq. (35) and Lemma 8] there holds the explicit formula

$$
z-\int_{-1}^{\hat{t}} \widehat{\mathrm{E}}^{r_{m}}(z) \mathrm{d} \widehat{\tau}=\frac{z}{2}\left(1-\hat{t}+\sum_{i=2}^{r_{m}+1}(-1)^{i}(2 i-1) \widehat{Q}_{i}(\hat{t})\right), \quad t \in \widehat{I},
$$

where

$$
\widehat{Q}_{i}(\hat{t})=\int_{-1}^{\hat{t}} \widehat{K}_{i-1}(\widehat{\tau}) \mathrm{d} \widehat{\tau}=\frac{\widehat{K}_{i}(\hat{t})-\widehat{K}_{i-2}(\hat{t})}{2 i-1}, \quad i \geq 2,
$$

with $\left\{\widehat{K}_{i}\right\}_{i \geq 0}$ signifying the family of Legendre polynomials on $(-1,1)$ (with degrees $\left.\operatorname{deg}\left(\widehat{K}_{i}\right)=i\right)$, scaled such that $\widehat{K}_{i}(-1)=(-1)^{i}$; cf. [15. Eq. (9) and Lemma 1]. Combining the above identities, we obtain

$$
z-\int_{-1}^{\hat{t}} \widehat{\mathrm{E}}^{r_{m}}(z) \mathrm{d} \widehat{\tau}=\frac{z}{2}\left(1-\hat{t}+\sum_{i=2}^{r_{m}+1}(-1)^{i}\left(\widehat{K}_{i}(\hat{t})-\widehat{K}_{i-2}(\hat{t})\right)\right) .
$$

Noticing the telescope sum as well as the fact that $\widehat{K}_{0}(\hat{t})=1$ and $\widehat{K}_{1}(\hat{t})=\hat{t}$, we arrive at

$$
z-\int_{-1}^{\hat{t}} \widehat{\mathrm{E}}^{r_{m}}(z) \mathrm{d} \widehat{\tau}=\frac{z}{2}(-1)^{r_{m}+1}\left(\widehat{K}_{r_{m}+1}(\hat{t})-\widehat{K}_{r_{m}}(\hat{t})\right)
$$

Then, employing the fact that

$$
\left|\widehat{K}_{i}(\hat{t})\right| \leq 1 \quad \forall \hat{t} \in[-1,1], \forall i \geq 0
$$

results in

$$
\left\|z-\int_{-1}^{\hat{t}} \widehat{\mathrm{E}}^{r_{m}}(z) \mathrm{d} \widehat{\tau}\right\|_{X} \leq\|z\|_{X} \quad \forall \hat{t} \in \widehat{I}
$$

Now we define the affine mapping

$$
F_{m}: \widehat{I} \rightarrow I_{m}, \quad \hat{t} \mapsto \frac{1}{2} k_{m} \hat{t}+\frac{1}{2}\left(t_{m-1}+t_{m}\right) .
$$

A scaling argument implies that

$$
\mathrm{E}_{m}^{r_{m}}(z) \circ F_{m}=\frac{2}{k_{m}} \widehat{\mathrm{E}}^{r_{m}}(z) ;
$$

see [15, Lemma 7]. Hence, by a change of variables, $\tau=F_{m}(\widehat{\tau}), \mathrm{d} \tau=\frac{k_{m}}{2} \mathrm{~d} \widehat{\tau}$, we conclude that

$$
\left\|z-\int_{t_{m-1}}^{t} \mathrm{E}_{m}^{r_{m}}(z) \mathrm{d} \tau\right\|_{X}=\left\|z-\int_{-1}^{F_{m}^{-1}(t)} \widehat{\mathrm{E}}^{r_{m}}(z) \mathrm{d} \widehat{\tau}\right\|_{X} \leq\|z\|_{X} \quad \forall t \in I_{m} .
$$

Noticing that, for $t=t_{m-1}$, there holds equality in the above bound, completes the proof. 
Lemma 2. Let $1 \leq m \leq M$, and $X$ a real Hilbert space. Then, the bound

$$
\left\|U_{m-1}^{+}\right\|_{X} \leq\|\chi(U)\|_{L^{1}\left(I_{m} ; X\right)}
$$

holds true for any $U \in \mathcal{P}^{r_{m}}\left(I_{m} ; X\right)$.

Proof. Let $U \in \mathcal{P}^{r_{m}}\left(I_{m} ; X\right)$. We define

$$
\Upsilon_{m}^{r_{m}}:=(-1)^{r_{m}} U_{m-1}^{+}\left(\widehat{K}_{r_{m}} \circ F_{m}^{-1}\right) \in \mathcal{P}^{r_{m}}\left(I_{m} ; X\right),
$$

where $\widehat{K}_{r_{m}}$ is the $r_{m}$-th Legendre polynomial on $(-1,1)$, which we scale such that $\widehat{K}_{r_{m}}(-1)=(-1)^{r_{m}}$ (cf. the proof of Lemma 1), and $F_{m}$ is the affine element mapping from (3.14). Then,

$$
\left\|\Upsilon_{m}^{r_{m}}\right\|_{L^{\infty}\left(I_{m} ; X\right)} \leq\left\|U_{m-1}^{+}\right\|_{X}\left\|\widehat{K}_{r_{m}} \circ F_{m}^{-1}\right\|_{L^{\infty}\left(I_{m}\right)} \leq\left\|U_{m-1}^{+}\right\|_{X}\left\|\widehat{K}_{r_{m}}\right\|_{L^{\infty}(-1,1)} .
$$

Involving (3.13) shows

$$
\left\|\Upsilon_{m}^{r_{m}}\right\|_{L^{\infty}\left(I_{m} ; X\right)} \leq\left\|U_{m-1}^{+}\right\|_{X}
$$

Furthermore, $\Upsilon_{m}^{r_{m}}$ is orthogonal to the space $\mathcal{P}^{r_{m}-1}\left(I_{m} ; X\right)$ (where $\mathcal{P}^{-1}\left(I_{m} ; X\right):=$ $\{0\} \subset X)$ with respect to the inner product in $L^{2}\left(I_{m} ; X\right)$. In particular, since $U^{\prime} \in$ $\mathcal{P}^{r_{m}-1}\left(I_{m} ; X\right)$, we have

$$
\int_{I_{m}}\left(U^{\prime}, \Upsilon_{m}^{r_{m}}\right)_{X} \mathrm{~d} t=0
$$

So, noticing that $\Upsilon_{m}^{r_{m}}\left(t_{m-1}^{+}\right)=U_{m-1}^{+}$, it follows that

$$
\begin{aligned}
\int_{I_{m}}\left(\chi(U), \Upsilon_{m}^{r_{m}}\right)_{X} \mathrm{~d} t & =\int_{I_{m}}\left(\mathrm{E}_{m}^{r_{m}}\left(U_{m-1}^{+}\right), \Upsilon_{m}^{r_{m}}\right)_{X} \mathrm{~d} t \\
& =\left(U_{m-1}^{+}, \Upsilon_{m}^{r_{m}}\left(t_{m-1}^{+}\right)\right)_{X}=\left\|U_{m-1}^{+}\right\|_{X}^{2}
\end{aligned}
$$

Therefore, using Hölder's inequality and recalling (3.16), we conclude that

$$
\left\|U_{m-1}^{+}\right\|_{X}^{2} \leq\|\chi(U)\|_{L^{1}\left(I_{m} ; X\right)}\left\|\Upsilon_{m}^{r_{m}}\right\|_{L^{\infty}\left(I_{m} ; X\right)} \leq\|\chi(U)\|_{L^{1}\left(I_{m} ; X\right)}\left\|U_{m-1}^{+}\right\|_{X} .
$$

Dividing by $\left\|U_{m-1}^{+}\right\|_{X}$ shows the desired bound.

We are now ready to show Proposition 11.

Proof of Proposition [1. Consider $U \in \mathcal{P}^{r_{m}}\left(I_{m} ; X\right)$. We choose $t^{\star} \in \bar{I}_{m}$ such that $\left\|U\left(t^{\star}\right)\right\|_{X}=$ $\|U\|_{L^{\infty}\left(I_{m} ; X\right)}$. It holds that

$$
U\left(t^{\star}\right)=\int_{t_{m-1}}^{t^{\star}}\left(U^{\prime}+\mathrm{E}_{m}^{r_{m}}\left(U_{m-1}^{+}\right)\right) \mathrm{d} \tau+U_{m-1}^{+}-\int_{t_{m-1}}^{t^{\star}} \mathrm{E}_{m}^{r_{m}}\left(U_{m-1}^{+}\right) \mathrm{d} \tau .
$$

Applying the triangle inequality as well as Bochner's Theorem, and recalling (3.12), this implies that

$$
\begin{aligned}
\|U\|_{L^{\infty}\left(I_{m} ; X\right)} & \leq \int_{t_{m-1}}^{t^{\star}}\|\chi(U)\|_{X} \mathrm{~d} \tau+\left\|U_{m-1}^{+}-\int_{t_{m-1}}^{t^{\star}} \mathrm{E}_{m}^{r_{m}}\left(U_{m-1}^{+}\right) \mathrm{d} \tau\right\|_{X} \\
& \leq\|\chi(U)\|_{L^{1}\left(I_{m} ; X\right)}+\left\|U_{m-1}^{+}\right\|_{X} .
\end{aligned}
$$

Inserting the bound (3.15) results in

$$
\|U\|_{L^{\infty}\left(I_{m} ; X\right)} \leq 2\|\chi(U)\|_{L^{1}\left(I_{m} ; X\right)},
$$

and applying Hölder's inequality completes the proof with $C_{\chi}=2$.

Remark 1. The proof of Proposition 1 reveals the upper bound $C_{\chi} \leq 2$. We emphasize, in particular, that the estimate (3.11) is uniform with respect to the local polynomial degree $r_{m} \geq 0$ as $r_{m} \rightarrow \infty$. 
Remark 2. Upon setting $U=\chi^{-1}(V)$ in (3.11), we obtain

$$
\left\|\chi^{-1}(V)\right\|_{L^{\infty}\left(I_{m} ; X\right)} \leq C_{\chi} k_{m}^{1-1 / p}\|V\|_{L^{p}\left(I_{m} ; X\right)},
$$

for any $V \in \mathcal{P}^{r_{m}}\left(I_{m} ; X\right)$.

3.2.2. Fixed Point Formulation and Existence of Discrete $d G$ Solutions. As for the $\mathrm{cG}$ method we prove the existence of solutions of (2.3) by means of a fixed point argument. For this purpose, we will derive a suitable fixed point formulation, and return to the case $X=H_{m}$. Noticing the fact that $\pi_{m} U_{m-1}^{+}=U_{m-1}^{+} \in H_{m}$, we observe that, on $I_{m}$, there holds

$$
\begin{aligned}
U^{\prime}+\mathrm{E}_{m}^{r_{m}}\left(\pi_{m} \llbracket U \rrbracket_{m-1}\right) & =\left(U-\pi_{m} U_{m-1}^{-}\right)^{\prime}+\mathrm{E}_{m}^{r_{m}}\left(U_{m-1}^{+}-\pi_{m} U_{m-1}^{-}\right) \\
& =\chi\left(U-\pi_{m} U_{m-1}^{-}\right),
\end{aligned}
$$

and recalling (3.8), we can write

$$
\chi\left(U-\pi_{m} U_{m-1}^{-}\right)=\Pi_{m}^{r_{m}} \mathcal{F}(U) .
$$

Applying Proposition 1 we infer that

$$
U=\pi_{m} U_{m-1}^{-}+\chi^{-1}\left(\Pi_{m}^{r_{m}} \mathcal{F}(U)\right) ;
$$

this is the 'dG-version' of the integral equation (3.2) for the cG method. Now, for given $U_{m-1}^{-}$(where as before $U_{0}^{-}:=u_{0}$ ) we define the operator

$$
\mathrm{T}_{m}^{\mathrm{dG}}: \mathcal{P}^{r_{m}}\left(I_{m} ; H_{m}\right) \rightarrow \mathcal{P}^{r_{m}}\left(I_{m} ; H_{m}\right)
$$

by

$$
\mathrm{T}_{m}^{\mathrm{dG}}(U):=\pi_{m} U_{m-1}^{-}+\chi^{-1}\left(\Pi_{m}^{r_{m}} \mathcal{F}(U)\right) .
$$

Then, $U \in \mathcal{P}^{r_{m}}\left(I_{m} ; H_{m}\right)$ solves (3.8) if and only if $U$ satisfies

$$
\mathrm{T}_{m}^{\mathrm{dG}}(U)=U
$$

We will now prove the existence of solutions to the local $h p$-dG time stepping scheme (2.3): Consider $\kappa_{m}, \theta_{m}>0$ (with $t_{m-1}+\theta_{m} \leq T$ ), and define the set

$$
Q_{m}=\left[t_{m-1}, t_{m-1}+\theta_{m}\right] \times B_{m},
$$

where

$$
B_{m}=\left\{y \in H_{m}:\left\|y-\pi_{m} U_{m-1}^{-}\right\|_{H} \leq \kappa_{m}\right\} .
$$

Due to the continuity of $\mathcal{F}$, its maximum on the compact set $Q_{m}$,

$$
K_{m}:=\max _{(t, y) \in Q_{m}}\|\mathcal{F}(t, y)\|_{H},
$$

exists. We choose

$$
0<k_{m} \leq \min \left(\theta_{m}, C_{\chi}^{-1} K_{m}^{-1} \kappa_{m}\right),
$$

where $C_{\chi}$ is the constant from (3.11), and introduce

$$
M_{m}^{\mathrm{dG}}:=\left\{Y \in \mathcal{P}^{r_{m}}\left(I_{m} ; H_{m}\right): Y(t) \in B_{m} \forall t \in \bar{I}_{m}\right\},
$$

with $I_{m}=\left(t_{m-1}, t_{m}\right), t_{m}=t_{m-1}+k_{m}$.

Consider any $U \in M_{m}^{\mathrm{dG}}$. From the definition of $\mathrm{T}_{m}^{\mathrm{dG}}$ in (3.18), and from (3.17) with $p=2$, we conclude that

$$
\left\|\mathrm{T}_{m}^{\mathrm{dG}}(U)-\pi_{m} U_{m-1}^{-}\right\|_{L^{\infty}\left(I_{m} ; H\right)} \leq C_{\chi} k_{m}^{1 / 2}\left\|\Pi_{m}^{r_{m}} \mathcal{F}(U)\right\|_{L^{2}\left(I_{m} ; H\right)} .
$$

The boundedness of the $L^{2}$-projection on $I_{m}$ (with constant 1 ) implies that

$$
\left\|\mathrm{T}_{m}^{\mathrm{dG}}(U)-\pi_{m} U_{m-1}^{-}\right\|_{L^{\infty}\left(I_{m} ; H\right)} \leq C_{\chi} k_{m}^{1 / 2}\|\mathcal{F}(U)\|_{L^{2}\left(I_{m} ; H\right)} .
$$

Then, we obtain

$$
\left\|\mathrm{T}_{m}^{\mathrm{dG}}(U)-\pi_{m} U_{m-1}^{-}\right\|_{L^{\infty}\left(I_{m} ; H\right)} \leq C_{\chi} k_{m}\|\mathcal{F}(U)\|_{L^{\infty}\left(I_{m} ; H\right)} \leq K_{m} C_{\chi} k_{m} \leq \kappa_{m},
$$


since $U \in M_{m}^{\mathrm{dG}}$. This implies that $\mathrm{T}_{m}^{\mathrm{dG}}\left(M_{m}^{\mathrm{dG}}\right) \subseteq M_{m}^{\mathrm{dG}}$. Then, employing Brower's fixed point theorem (based on the fact that $M_{m}^{\mathrm{dG}}$ is convex and compact, and that $\mathrm{T}_{m}^{\mathrm{dG}}$ is continuous), there exists a solution of (3.19), and therefore of (3.8) and (2.3).

\section{Uniqueness of Galerkin Solutions}

In order to obtain unique Galerkin solutions on each time step we apply a contraction argument following the approach presented in 2. To this end, we make the assumption that the nonlinearity $\mathcal{F}$ is locally Lipschitz continuous. Then, if the local time step $k_{m}$ in the Galerkin time discretizations is chosen sufficiently small (again, independently of the local polynomial degree), we will show that the operators $\mathrm{T}_{m}^{\mathrm{cG}}$ and $\mathrm{T}_{m}^{\mathrm{dG}}$ from (3.3) and (3.18), respectively, are contractive. This will lead to the following uniqueness result.

Theorem 2. Let $m \geq 1$, and $\kappa_{m}, \theta_{m}>0$ (with $t_{m-1}+\theta_{m} \leq T$ ). Furthermore, consider $B_{m}$ from (3.5), $K_{m}$ from (3.6), and $M_{m}^{\mathrm{cG}}$ from (3.7) for the $c G$ method (2.2), and the respective quantities for the $d G$ scheme (2.3) from (3.20), (3.21), and (3.23). Moreover, for each of the two schemes, we suppose that there exists a constant $0 \leq L_{\mathcal{F}}\left(B_{m}\right)<\infty$ such that the local Lipschitz continuity condition,

$$
\|\mathcal{F}(t, u)-\mathcal{F}(t, v)\|_{H} \leq L_{\mathcal{F}}\left(B_{m}\right)\|u-v\|_{H} \quad \forall t \in \bar{I}_{m}, \forall u, v \in B_{m},
$$

holds. In addition, for a parameter $\varrho \in(0,1)$, suppose that

$$
k_{m} \leq \min \left(\theta_{m}, c^{-1} K_{m}^{-1} \kappa_{m}, \varrho c^{-1} L_{\mathcal{F}}\left(B_{m}\right)^{-1}\right),
$$

where

$$
c= \begin{cases}1 & \text { for } c G \text { time stepping }, \\ C_{\chi} & \text { for } d G \text { time stepping },\end{cases}
$$

with $C_{\chi}$ being the constant from (3.11). Then, the $c G$ and $d G$ methods on $I_{m}$ each possess unique solutions $U_{\mathrm{cG}}$ and $U_{\mathrm{dG}}$ in $M_{m}^{\mathrm{cG}}$ and $M_{m}^{\mathrm{dG}}$, respectively.

Proof. We treat the cG and dG cases separately.

Uniqueness of $c G$ solution: From Section 3.1 we recall the following fact: For given $\kappa_{m}, \theta_{m}>0$ (with $t_{m-1}+\theta_{m} \leq T$ ), and for $K_{m}$ from (3.6), choosing the local time step $k_{m}$ to be bounded by $k_{m} \leq \min \left(\theta_{m}, K_{m}^{-1} \kappa_{m}\right)$ guarantees the self-mapping property $\mathrm{T}_{m}^{c G}\left(M_{m}^{c G}\right) \subseteq M_{m}^{c G}$, where $\mathrm{T}_{m}^{c G}$ is the cG operator from (3.3). Furthermore, for $U_{1}, U_{2} \in M_{m}^{\mathrm{cG}}$ we have

$$
\begin{aligned}
\left\|\mathrm{T}_{m}^{\mathrm{cG}}\left(U_{1}\right)-\mathrm{T}_{m}^{\mathrm{cG}}\left(U_{2}\right)\right\|_{L^{\infty}\left(I_{m} ; H\right)} & =\left\|\int_{t_{m-1}}^{t} \Pi_{m}^{r_{m}}\left(\mathcal{F}\left(U_{1}\right)-\mathcal{F}\left(U_{2}\right)\right) \mathrm{d} \tau\right\|_{L^{\infty}\left(I_{m} ; H\right)} \\
& \leq \int_{I_{m}}\left\|\Pi_{m}^{r_{m}}\left(\mathcal{F}\left(U_{1}\right)-\mathcal{F}\left(U_{2}\right)\right)\right\|_{H} \mathrm{~d} \tau \\
& \leq k_{m}^{1 / 2}\left\|\Pi_{m}^{r_{m}}\left(\mathcal{F}\left(U_{1}\right)-\mathcal{F}\left(U_{2}\right)\right)\right\|_{L^{2}\left(I_{m} ; H\right)} \\
& \leq k_{m}^{1 / 2}\left\|\mathcal{F}\left(U_{1}\right)-\mathcal{F}\left(U_{2}\right)\right\|_{L^{2}\left(I_{m} ; H\right)} \\
& \leq k_{m}\left\|\mathcal{F}\left(U_{1}\right)-\mathcal{F}\left(U_{2}\right)\right\|_{L^{\infty}\left(I_{m} ; H\right)} .
\end{aligned}
$$

Now involving the Lipschitz condition (4.1) on $B_{m}$ from (3.5), we infer that

$$
\left\|\mathrm{T}_{m}^{\mathrm{cG}}\left(U_{1}\right)-\mathrm{T}_{m}^{\mathrm{cG}}\left(U_{2}\right)\right\|_{L^{\infty}\left(I_{m} ; H\right)} \leq L_{\mathcal{F}}\left(B_{m}\right) k_{m}\left\|U_{1}-U_{2}\right\|_{L^{\infty}\left(I_{m} ; H\right)},
$$

for all $U_{1}, U_{2} \in M_{m}^{\mathrm{cG}}$. This implies that, for $k_{m}<L_{\mathcal{F}}\left(B_{m}\right)^{-1}$, the operator $\mathrm{T}_{m}^{\mathrm{cG}}$ is a contraction on $M_{m}^{\mathrm{cG}}$. Thus, by the Banach fixed point theorem, the equation (3.4) has a unique solution in $M_{m}^{\mathrm{cG}}$. 
Uniqueness of $d G$ solution: In the case of the $\mathrm{dG}$ scheme we proceed in a similar way as for the cG time stepping method. For $\kappa_{m}, \theta_{m}>0$ (with $\left.t_{m-1}+\theta_{m} \leq T\right)$, and for $K_{m}$ from (3.21), choosing the local time step $k_{m}$ to be bounded by $k_{m} \leq$ $\min \left(\theta_{m}, C_{\chi}^{-1} K_{m}^{-1} \kappa_{m}\right)$ ensures that $\mathrm{T}_{m}^{\mathrm{dG}}\left(M_{m}^{\mathrm{dG}}\right) \subseteq M_{m}^{\mathrm{dG}}$, where $C_{\chi}$ is the constant from (3.11), and $\mathrm{T}_{m}^{\mathrm{dG}}$ is the operator defined in (3.18); cf. Section 3.2.2. In addition, for $U_{1}, U_{2} \in M_{m}^{\mathrm{dG}}$ there holds that

$$
\left\|\mathrm{T}_{m}^{\mathrm{dG}}\left(U_{1}\right)-\mathrm{T}_{m}^{\mathrm{dG}}\left(U_{2}\right)\right\|_{L^{\infty}\left(I_{m} ; H\right)}=\left\|\chi^{-1}\left(\Pi_{m}^{r_{m}} \mathcal{F}\left(U_{1}\right)-\Pi_{m}^{r_{m}} \mathcal{F}\left(U_{2}\right)\right)\right\|_{L^{\infty}\left(I_{m} ; H\right)} .
$$

Using (3.17), we deduce that

$$
\begin{aligned}
\left\|\mathrm{T}_{m}^{\mathrm{dG}}\left(U_{1}\right)-\mathrm{T}_{m}^{\mathrm{dG}}\left(U_{2}\right)\right\|_{L^{\infty}\left(I_{m} ; H\right)} & \leq C_{\chi} k_{m}^{1 / 2}\left\|\Pi_{m}^{r_{m}}\left(\mathcal{F}\left(U_{1}\right)-\mathcal{F}\left(U_{2}\right)\right)\right\|_{L^{2}\left(I_{m} ; H\right)} \\
& \leq C_{\chi} k_{m}^{1 / 2}\left\|\mathcal{F}\left(U_{1}\right)-\mathcal{F}\left(U_{2}\right)\right\|_{L^{2}\left(I_{m} ; H\right)} \\
& \leq C_{\chi} k_{m}\left\|\mathcal{F}\left(U_{1}\right)-\mathcal{F}\left(U_{2}\right)\right\|_{L^{\infty}\left(I_{m} ; H\right)} .
\end{aligned}
$$

By means of (4.1) we derive the bound

$$
\left\|\mathrm{T}_{m}^{\mathrm{dG}}\left(U_{1}\right)-\mathrm{T}_{m}^{\mathrm{dG}}\left(U_{2}\right)\right\|_{L^{\infty}\left(I_{m} ; H\right)} \leq C_{\chi} L_{\mathcal{F}}\left(B_{m}\right) k_{m}\left\|U_{1}-U_{2}\right\|_{L^{\infty}\left(I_{m} ; H\right)},
$$

for all $U_{1}, U_{2} \in M_{m}^{\mathrm{dG}}$, where the ball $B_{m}$ is defined in (3.20). Hence, for $k_{m}<$ $C_{\chi}^{-1} L_{\mathcal{F}}\left(B_{m}\right)^{-1}$, the mapping $\mathrm{T}_{m}^{\mathrm{dG}}: M_{m}^{\mathrm{dG}} \rightarrow M_{m}^{\mathrm{dG}}$ is a contraction. This implies that the equation (3.19) has a unique solution $U \in M_{m}^{\mathrm{dG}}$.

Remark 3. The above Theorem 2 shows that the $\mathrm{cG}$ and $\mathrm{dG}$ operators in (3.3) and (3.18) are contractions in each time step, and thus, have unique fixed points in $M_{m}^{\mathrm{cG}}$ and $M_{m}^{\mathrm{dG}}$, respectively. In particular, the corresponding fixed point iterations converge. For instance, in the case of the $\mathrm{cG}$ time stepping scheme, for $m \geq 1$, starting from an initial guess $U^{(0)} \in M_{m}^{\mathrm{cG}}$ (which can be chosen, for example, to be the constant function $\left.U^{(0)}(t)=\pi_{m} U_{m-1}, t \in I_{m}\right)$, the iteration

$$
U^{(\ell+1)}=\mathrm{T}_{m}^{\mathrm{cG}}\left(U^{(\ell)}\right), \quad \ell \geq 1,
$$

will tend to the unique solution $\left.U\right|_{I_{m}} \in M_{m}^{\mathrm{cG}}$ of (2.2). Similarly, for the dG scheme, for $m \geq 1$, and an initial guess $U^{(0)} \in M_{m}^{\mathrm{dG}}$ (for example, $U^{(0)}(t)=\pi_{m} U_{m-1}^{-}$, $\left.t \in I_{m}\right)$, the iteration

$$
U^{(\ell+1)}=\mathrm{T}_{m}^{\mathrm{dG}}\left(U^{(\ell)}\right), \quad \ell \geq 1,
$$

converges to the unique solution $\left.U\right|_{I_{m}} \in M_{m}^{\mathrm{dG}}$ of $(\underline{2.3})$.

\section{Applichtion to Finite-Time Blow-Up Problems}

In this section we will discuss the existence and uniqueness Theorem 2 in the context of nonlinearities $\mathcal{F}$ that grow algebraically with respect to $u$, with a power larger than 1. We will show that both the exact solution $u$ of (1.1) as well as the $\mathrm{cG}$ and $\mathrm{dG}$ solutions blow up in finite time. In addition, we will provide a time step selection algorithm, and prove a convergence result. In order to keep the technical matters within a reasonable scope, we assume that $H$ is finite dimensional, and that $H=H_{1}=H_{2}=\ldots=H_{m}=\ldots$ holds for any $m \geq 1$.

5.1. Algebraic Growth Nonlinearities. We consider nonlinearities $\mathcal{F}$ which feature the following algebraic growth condition: Suppose that there exist constants $\alpha, \delta>0, \beta>1$, and $c_{\mathcal{F}} \geq 0$ such that

$$
\|\mathcal{F}(t, u)\|_{H} \leq \alpha\|u\|_{H}^{\beta} \quad \text { and } \quad(\mathcal{F}(t, u), u)_{H} \geq \delta\|u\|_{H}^{1+\beta},
$$

for all $u \in H$ which satisfy $\|u\|_{H} \geq c_{\mathcal{F}}$, and for any $t \in[0, \infty$ ) (or for any $t \in[0, T]$, with sufficiently large $T>0$ ). We note that such problems exhibit a blow-up in some finite time $T_{\infty}<\infty$. Indeed, let $u$ solve (1.1), and suppose that $\left\|u_{0}\right\|_{H}>c_{\mathcal{F}}$ 
in (1.1). Then, under the conditions (5.1), it is easy to see that $\|u(t)\|_{H}$ is nondecreasing with respect to $t$, and thus,

$$
\frac{\mathrm{d}}{\mathrm{d} t}\|u(t)\|_{H}^{2}=2\left(u^{\prime}(t), u(t)\right)_{H}=2(\mathcal{F}(t, u(t)), u(t))_{H} \geq 2 \delta\left(\|u(t)\|_{H}^{2}\right)^{(1+\beta) / 2} .
$$

Hence,

$$
\frac{1}{1-\beta} \frac{\mathrm{d}}{\mathrm{d} t}\left[\left(\|u(t)\|_{H}^{2}\right)^{(1-\beta) / 2}\right] \geq \delta
$$

Integrating from 0 to some $t>0$ shows that

$$
\left\|u_{0}\right\|_{H}^{1-\beta}-\|u(t)\|_{H}^{1-\beta} \geq(\beta-1) \delta t
$$

and therefore,

$$
t \leq \frac{\left\|u_{0}\right\|_{H}^{1-\beta}}{(\beta-1) \delta}=: \bar{T}_{\infty}
$$

It follows that $\bar{T}_{\infty}$ is an upper bound for the blow-up time.

5.2. Discrete Blow-Up. Provided that the properties (5.1) hold true, the goal of this section is to show that the $\mathrm{cG}$ and $\mathrm{dG}$ time stepping methods yield solutions which blow-up in finite time. To this end, let us assume, in addition to (5.1), that the local Lipschitz property

$$
\|\mathcal{F}(t, u)-\mathcal{F}(t, v)\|_{H} \leq \gamma \max \left(\|u\|_{H},\|v\|_{H}\right)^{\beta-1}\|u-v\|_{H}
$$

holds true whenever $\|u\|_{H},\|v\|_{H} \geq c_{\mathcal{F}}$, cf. (5.1), and for all $t \in[0, \infty$ ) (or for any $t \in[0, T]$ with sufficiently large $T>0)$, with a uniform constant $\gamma \geq 0$.

In the following elaborations, the function

$$
\Psi:[0, \gamma / \alpha) \rightarrow \mathbb{R}, \quad \varrho \mapsto \Psi(\varrho)=\frac{\delta(\gamma-\varrho \alpha)^{\beta}-\varrho \alpha \gamma^{\beta}}{\gamma-\varrho \alpha}
$$

will play an important role; here, $\alpha, \beta, \delta$, and $\gamma$ are the constants from (5.1) and (5.3), respectively. We note that $\Psi$ is decreasing, and that $\Psi(0)=\delta \gamma^{\beta-1}>0$, and $\lim _{\varrho \nearrow \gamma / \alpha} \Psi(\varrho)=-\infty$. Hence, by continuity there exists exactly one zero $\varrho$ of $\Psi$ in the interval $[0, \gamma / \alpha)$.

Proposition 2. Suppose that the conditions (5.1) and (5.3) hold, and that the initial value $u_{0} \in H$ from (1.1) satisfies $\left\|u_{0}\right\|_{H}>c_{\mathcal{F}}$. Furthermore, let $\varrho_{0}$ be a fixed constant with $0<\varrho_{0}<\min (1, \bar{\varrho})$, where $\bar{\varrho}$ is the unique zero of $\Psi$ from (5.4) in $[0, \gamma / \alpha)$. For any given $\varrho$ with

$$
0<\varrho \leq \min \left(\varrho_{0}, \frac{\alpha^{-1} \gamma}{1+\left(1-c_{\mathcal{F}}\left\|u_{0}\right\|_{H}^{-1}\right)^{-1}}\right),
$$

choose the time steps to be

$$
k_{m}(\varrho):=c^{-1} \gamma^{-\beta} \varrho(\gamma-\varrho \alpha)^{\beta-1}\left\|U_{m-1}^{-}\right\|_{H}^{1-\beta}, \quad m=1,2,3, \ldots,
$$

where $U_{m-1}^{-}, m \geq 1$, signifies the left-sided value of the $c G$ or $d G$ solution $U$ from (2.2) or (2.3), respectively, at the nodal point $t_{m-1}$ (with $U_{m-1}^{-}=U_{m-1}$ for the $c G$ scheme, and $\left.U_{0}^{-}:=u_{0}\right)$. Then, there holds:

(i) For any $m \geq 1$, the $c G$ and $d G$ solutions resulting from (2.2) and (2.3) exist and are unique in $M_{m}^{\mathrm{cG}}$ from (3.7) and $M_{m}^{\mathrm{dG}}$ from (3.23), respectively, with $\kappa_{m}=\varrho \alpha(\gamma-\varrho \alpha)^{-1}\left\|U_{m-1}^{-}\right\|_{H}$, for any polynomial degree distribution.

(ii) Both the $c G$ and the $d G$ solutions blow-up at finite times $\widetilde{T}_{\infty}^{\mathrm{cG}}(\varrho)$ and $\widetilde{T}_{\infty}^{\mathrm{dG}}(\varrho)$, respectively.

The constants $\alpha, \beta, \delta$, and $\gamma$ were introduced in (5.1) and (5.3), respectively, and $c$ is defined in (4.3). 
Proof. We focus on the dG method only; the proof for the cG method can be done verbatim. Let $m \geq 1$, and suppose that the $\mathrm{dG}$ solution on the first $m-1$ time steps is well-defined, and that

$$
\left\|U_{m-1}^{-}\right\|_{H} \geq\left\|u_{0}\right\|_{H}>c_{\mathcal{F}} \geq 0 .
$$

Then, with $\kappa_{m}=\eta_{m}\left\|U_{m-1}^{-}\right\|_{H}$, where

$$
\eta_{m}=\varrho \alpha(\gamma-\varrho \alpha)^{-1},
$$

we see by means of (5.5) that $0<\eta_{m} \leq 1-c_{\mathcal{F}}\left\|u_{0}\right\|_{H}^{-1}$. Therefore, for any $y \in$ $B_{m}:=\left\{y \in H:\left\|y-U_{m-1}^{-}\right\|_{H} \leq \kappa_{m}\right\}$, it follows that

$$
\begin{aligned}
\|y\|_{H} & \geq\left\|U_{m-1}^{-}\right\|_{H}-\left\|y-U_{m-1}^{-}\right\|_{H} \geq\left\|U_{m-1}^{-}\right\|_{H}-\kappa_{m} \geq\left(1-\eta_{m}\right)\left\|U_{m-1}^{-}\right\|_{H} \\
& \geq\left(1-\eta_{m}\right)\left\|u_{0}\right\|_{H} \geq c_{\mathcal{F}} .
\end{aligned}
$$

Consequently, in view of the growth condition (5.1), there holds

$$
\begin{aligned}
K_{m}: & =\max _{(t, y) \in Q_{m}}\|\mathcal{F}(t, y)\|_{H} \\
& \leq \alpha\|y\|_{H}^{\beta} \leq \alpha\left(\left\|U_{m-1}^{-}\right\|_{H}+\kappa_{m}\right)^{\beta}=\alpha\left(1+\eta_{m}\right)^{\beta}\left\|U_{m-1}^{-}\right\|_{H}^{\beta},
\end{aligned}
$$

with $Q_{m}=I_{m} \times B_{m}$, where $I_{m}=\left[t_{m-1}, t_{m-1}+\theta_{m}\right]$, and $\theta_{m}:=k_{m}(\varrho)$. Hence,

$$
k_{m}(\varrho)=c^{-1} \alpha^{-1} \eta_{m}\left(1+\eta_{m}\right)^{-\beta}\left\|U_{m-1}^{-}\right\|_{H}^{1-\beta} \leq c^{-1} K_{m}^{-1} \kappa_{m},
$$

and revisiting the existence proof in Section 3.2.2 (in particular, see (3.22) ), we infer that there is a $\mathrm{dG}$ solution in $M_{m}^{\mathrm{dG}}$. Furthermore, we bound the Lipschitz constant $L_{\mathcal{F}}\left(B_{m}\right)$ appearing in (4.1) by means of (5.3): For any $u, v \in B_{m}$ we have $\|u\|_{H},\|v\|_{H} \geq c_{\mathcal{F}}$ as shown before, and $\max \left(\|u\|_{H},\|v\|_{H}\right) \leq\left\|U_{m-1}^{-}\right\|_{H}+\kappa_{m}$. Thus,

$$
L_{\mathcal{F}}\left(B_{m}\right) \leq \gamma\left(\kappa_{m}+\left\|U_{m-1}^{-}\right\|_{H}\right)^{\beta-1} \leq \gamma\left(1+\eta_{m}\right)^{\beta-1}\left\|U_{m-1}^{-}\right\|_{H}^{\beta-1},
$$

which implies that

$$
k_{m}(\varrho)=\varrho c^{-1} \gamma^{-1}\left(1+\eta_{m}\right)^{1-\beta}\left\|U_{m-1}^{-}\right\|_{H}^{1-\beta} \leq \varrho c^{-1} L_{\mathcal{F}}\left(B_{m}\right)^{-1} .
$$

Then, with reference to (4.2), the uniqueness of a dG solution in $M_{m}^{\mathrm{dG}}$ follows immediately.

Next, consider the dG solution $\left.U\right|_{I_{m}} \in \mathcal{P}^{r_{m}}\left(I_{m} ; H\right)$. Using (2.3) with the constant test function $V(t)=U_{m-1}^{-}, t \in I_{m}$, we have that

$$
\left(U_{m}^{-}, U_{m-1}^{-}\right)_{H}=\left\|U_{m-1}^{-}\right\|_{H}^{2}+\int_{I_{m}}\left(\mathcal{F}(U), U_{m-1}^{-}\right)_{H} \mathrm{~d} t .
$$

Recalling (5.7), and employing (5.1), we obtain

$$
\begin{aligned}
& \left\|U_{m}^{-}\right\|_{H}\left\|U_{m-1}^{-}\right\|_{H} \\
& \quad \geq\left\|U_{m-1}^{-}\right\|_{H}^{2}+k_{m}\left(\mathcal{F}\left(U_{m-1}^{-}\right), U_{m-1}^{-}\right)_{H}+\int_{I_{m}}\left(\mathcal{F}(U)-\mathcal{F}\left(U_{m-1}^{-}\right), U_{m-1}^{-}\right)_{H} \mathrm{~d} t \\
& \quad \geq\left\|U_{m-1}^{-}\right\|_{H}^{2}+k_{m} \delta\left\|U_{m-1}^{-}\right\|_{H}^{1+\beta}-\left\|U_{m-1}^{-}\right\|_{H} \int_{I_{m}}\left\|\mathcal{F}(U)-\mathcal{F}\left(U_{m-1}^{-}\right)\right\|_{H} \mathrm{~d} t .
\end{aligned}
$$

Furthermore, dividing by $\left\|U_{m-1}^{-}\right\|_{H}>0$, it holds that

$$
\left\|U_{m}^{-}\right\|_{H} \geq\left\|U_{m-1}^{-}\right\|_{H}+k_{m} \delta\left\|U_{m-1}^{-}\right\|_{H}^{\beta}-\int_{I_{m}}\left\|\mathcal{F}(U)-\mathcal{F}\left(U_{m-1}^{-}\right)\right\|_{H} \mathrm{~d} t .
$$


Reviewing the proof of Theorem 1 in Section 3.2.2 we observe that $U(t) \in B_{m}$ for all $t \in I_{m}$. Therefore, using the local Lipschitz continuity (5.3) with the bound (5.9), it follows that

$$
\begin{aligned}
\left\|U_{m}^{-}\right\|_{H} & \geq\left\|U_{m-1}^{-}\right\|_{H}+k_{m} \delta\left\|U_{m-1}^{-}\right\|_{H}^{\beta}-k_{m} L_{\mathcal{F}}\left(B_{m}\right) \kappa_{m} \\
& \geq\left\|U_{m-1}^{-}\right\|_{H}+k_{m}\left(\delta-\gamma \eta_{m}\left(1+\eta_{m}\right)^{\beta-1}\right)\left\|U_{m-1}^{-}\right\|_{H}^{\beta} .
\end{aligned}
$$

Inserting (5.8) yields

$$
\left\|U_{m}^{-}\right\|_{H} \geq\left\|U_{m-1}^{-}\right\|_{H}+k_{m}\left(\delta-\varrho \alpha \gamma^{\beta}(\gamma-\varrho \alpha)^{-\beta}\right)\left\|U_{m-1}^{-}\right\|_{H}^{\beta} .
$$

Then, employing (5.6), and recalling that $\Psi$ is monotone decreasing, leads to

$$
\left\|U_{m}^{-}\right\|_{H} \geq\left(1+c^{-1} \gamma^{-\beta} \varrho \Psi(\varrho)\right)\left\|U_{m-1}^{-}\right\|_{H} \geq\left(1+C_{0} \varrho\right)\left\|U_{m-1}^{-}\right\|_{H},
$$

with

$$
C_{0}=c^{-1} \gamma^{-\beta} \Psi\left(\varrho_{0}\right) .
$$

The assumption (5.7) is trivially valid for $m=1$. Furthermore, due to (5.10) we note the fact that $\left\|U_{1}^{-}\right\|_{H} \geq\left\|u_{0}\right\|_{H}$, and, thus, we conclude inductively that the previous derivations are applicable for any $m \geq 2$.

Moreover, from (5.10) we infer that

$$
\left\|U_{m-1}^{-}\right\|_{H} \geq\left(1+C_{0} \varrho\right)^{m-k}\left\|U_{k-1}^{-}\right\|_{H} \quad \forall m \geq k \geq 1,
$$

which shows that $\left\|U_{m-1}^{-}\right\|_{H} \rightarrow \infty$ as $m \rightarrow \infty$. In addition, involving (5.6) it follows, for any $m \geq i \geq 1$, that

$$
\begin{aligned}
t_{m} & =t_{i-1}+\sum_{j=i}^{m} k_{j}(\varrho)=t_{i-1}+\frac{\varrho(\gamma-\alpha \varrho)^{\beta-1}}{c \gamma^{\beta}} \sum_{j=i}^{m}\left\|U_{j-1}^{-}\right\|_{H}^{1-\beta} \\
& \leq t_{i-1}+\frac{\varrho(\gamma-\alpha \varrho)^{\beta-1}}{c \gamma^{\beta}}\left\|U_{i-1}^{-}\right\|_{H}^{1-\beta} \sum_{j=i}^{m}\left(1+C_{0} \varrho\right)^{(1-\beta)(j-i)} \\
& \leq t_{i-1}+\frac{\varrho(\gamma-\alpha \varrho)^{\beta-1}}{c \gamma^{\beta}}\left\|U_{i-1}^{-}\right\|_{H}^{1-\beta} \sum_{j=0}^{\infty}\left(1+C_{0} \varrho\right)^{(1-\beta) j} .
\end{aligned}
$$

Therefore,

$$
t_{m} \leq t_{i-1}+\frac{\varrho(\gamma-\alpha \varrho)^{\beta-1}}{c \gamma^{\beta}} \frac{\left\|U_{i-1}^{-}\right\|_{H}^{1-\beta}}{1-\left(1+C_{0} \varrho\right)^{1-\beta}}, \quad m \geq i \geq 1 .
$$

In particular, for $i=1$ and $m \rightarrow \infty$, we see that the discrete blow-up time $\widetilde{T}_{\infty}^{\mathrm{dG}}(\varrho)$ for the $\mathrm{dG}$ method is bounded by

$$
\widetilde{T}_{\infty}^{\mathrm{dG}}(\varrho) \leq \frac{\varrho(\gamma-\alpha \varrho)^{\beta-1}}{c \gamma^{\beta}} \frac{\left\|u_{0}\right\|_{H}^{1-\beta}}{1-\left(1+C_{0} \varrho\right)^{1-\beta}}<\infty .
$$

This concludes the proof.

Remark 4. The above proof allows to establish an $L^{\infty}$ bound on the cG and dG solution, again denoted by $U$, on $\left(0, t_{m}\right)$, for $m \geq 1$. Indeed, for any $1 \leq i \leq m$, using (5.8), we have that

$$
\|U\|_{L^{\infty}\left(I_{i} ; H\right)} \leq\left\|U_{i-1}^{-}\right\|_{H}+\kappa_{i} \leq\left(1+\eta_{i}\right)\left\|U_{i-1}^{-}\right\|_{H} \leq \varsigma\left\|U_{i-1}^{-}\right\|_{H} \leq \varsigma\left\|U_{m-1}^{-}\right\|_{H},
$$

with $\varsigma=\gamma\left(\gamma-\varrho_{0} \alpha\right)^{-1}$. Taking the maximum for all $1 \leq i \leq m$, we conclude that $\|U\|_{L^{\infty}\left(\left(0, t_{m}\right) ; H\right)} \leq \varsigma\left\|U_{m-1}^{-}\right\|_{H}$. 
Remark 5. We notice that

$$
\mu:=\lim _{\varrho \searrow 0} \frac{\varrho(\gamma-\alpha \varrho)^{\beta-1}}{c \gamma^{\beta}\left(1-\left(1+C_{0} \varrho\right)^{1-\beta}\right)}=\frac{1}{c \gamma C_{0}(\beta-1)}>0
$$

in (5.14). In particular, we see that the discrete blow-up times $\widetilde{T}_{\infty}^{\mathrm{cG}}(\varrho)$ and $\widetilde{T}_{\infty}^{\mathrm{dG}}(\varrho)$ for the cG and dG methods, respectively, are uniformly bounded for any $\varrho$ satisfying (5.5).

Remark 6. Using (5.1), we can show that there exists a constant $C_{1}>0$, with $C_{1} \geq$ $C_{0}$ from (5.11), such that

$$
\left\|U_{m}^{-}\right\|_{H} \leq\left(1+C_{1} \varrho\right)\left\|U_{m-1}^{-}\right\|_{H}, \quad m \geq 1,
$$

for both the $\mathrm{cG}$ and $\mathrm{dG}$ solutions. To see this, consider, for instance, the $\mathrm{dG}$ solution $\left.U\right|_{I_{m}} \in \mathcal{P}^{r_{m}}\left(I_{m} ; H\right)$. Applying (2.3) with the constant test function $V(t)=$ $U_{m}^{-}, t \in I_{m}$, yields

$$
\left\|U_{m}^{-}\right\|_{H}^{2}=\left(U_{m-1}^{-}, U_{m}^{-}\right)_{H}+\int_{I_{m}}\left(\mathcal{F}(U), U_{m}^{-}\right)_{H} \mathrm{~d} t .
$$

Then, proceeding as in the proof of Proposition 2, there holds

$$
\begin{aligned}
\left\|U_{m}^{-}\right\|_{H}^{2}= & \left(U_{m-1}^{-}, U_{m}^{-}\right)_{H}+k_{m}\left(\mathcal{F}\left(U_{m-1}^{-}\right), U_{m}^{-}\right)_{H}+\int_{I_{m}}\left(\mathcal{F}(U)-\mathcal{F}\left(U_{m-1}^{-}\right), U_{m}^{-}\right)_{H} \mathrm{~d} t \\
\leq & \left\|U_{m-1}^{-}\right\|_{H}\left\|U_{m}^{-}\right\|_{H}+k_{m}\left\|\mathcal{F}\left(U_{m-1}^{-}\right)\right\|_{H}\left\|U_{m}^{-}\right\|_{H} \\
& +\left\|U_{m}^{-}\right\|_{H} \int_{I_{m}}\left\|\mathcal{F}(U)-\mathcal{F}\left(U_{m-1}^{-}\right)\right\|_{H} \mathrm{~d} t .
\end{aligned}
$$

Dividing by $\left\|U_{m}^{-}\right\|_{H}$, involving (5.1), (5.3), and (5.9), and recalling that $\| U$ $U_{m-1}^{-} \|_{L^{\infty}\left(I_{m} ; H\right)} \leq \kappa_{m}$, we infer

$$
\begin{aligned}
\left\|U_{m}^{-}\right\|_{H} & \leq\left\|U_{m-1}^{-}\right\|_{H}+k_{m} \alpha\left\|U_{m-1}^{-}\right\|_{H}^{\beta}+k_{m} L_{\mathcal{F}}\left(B_{m}\right) \kappa_{m} \\
& \leq\left(1+k_{m}\left(\alpha+\gamma \eta_{m}\left(1+\eta_{m}\right)^{\beta-1}\right)\left\|U_{m-1}^{-}\right\|_{H}^{\beta-1}\right)\left\|U_{m-1}^{-}\right\|_{H} .
\end{aligned}
$$

Inserting (5.6) and (5.8) we arrive at

$$
\left\|U_{m}^{-}\right\|_{H} \leq\left(1+\frac{\varrho \alpha\left(\varrho \gamma^{\beta}+(\gamma-\varrho \alpha)^{\beta}\right)}{c \gamma^{\beta}(\gamma-\varrho \alpha)}\right)\left\|U_{m-1}^{-}\right\|_{H} \leq\left(1+C_{1} \varrho\right)\left\|U_{m-1}^{-}\right\|_{H},
$$

with

$$
C_{1}=\alpha c^{-1}\left(\varrho_{0}+1\right)\left(\gamma-\varrho_{0} \alpha\right)^{-1}
$$

Proceeding analogously for the $\mathrm{cG}$ method, precisely the same bound can be proved. Moreover, in analogy to the derivation of (5.13), the bounds

$$
\begin{aligned}
t_{m} & \geq t_{i-1}+\frac{\varrho(\gamma-\alpha \varrho)^{\beta-1}}{c \gamma^{\beta}}\left\|U_{i-1}^{-}\right\|_{H}^{1-\beta} \sum_{j=i}^{m}\left(1+C_{1} \varrho\right)^{(1-\beta)(j-i)} \\
& \geq t_{i-1}+\frac{\varrho(\gamma-\alpha \varrho)^{\beta-1}}{c \gamma^{\beta}}\left\|U_{i-1}^{-}\right\|_{H}^{1-\beta} \sum_{j=0}^{m-i}\left(1+C_{1} \varrho\right)^{(1-\beta) j}
\end{aligned}
$$

for any $m \geq i \geq 1$, are obtained. 
5.3. Convergence to Blow-Up Time. We will now show that the cG and dG time stepping schemes are able to approximate the exact blow-up time as $\varrho \searrow 0$ in (5.6). To this end, we first establish a few auxiliary results.

Lemma 3. Suppose that the assumptions of Proposition 2 are fulfilled; in particular choose $\varrho$ as in (5.5), and let the time steps $\left\{k_{m}(\varrho)\right\}_{m \geq 1}$ be given by (5.6). In addition to the local Lipschitz property (5.3), suppose that there exists a constant $L_{c_{\mathcal{F}}}$ such that

$$
\|\mathcal{F}(u, t)-\mathcal{F}(v, t)\|_{H} \leq L_{c_{\mathcal{F}}}\|u-v\|_{H} \quad \forall t \in[0, \infty),
$$

whenever $\|u\|_{H},\|v\|_{H} \leq c_{\mathcal{F}}$. Furthermore, let $T_{0}>0$ be fixed with

$$
T_{0}<\min \left(T_{\infty}(\varrho), \widetilde{T}_{\infty}(\varrho)\right)<\infty
$$

where $T_{\infty}(\varrho)$ and $\widetilde{T}_{\infty}(\varrho)$ are the exact and the discrete blow-up times (i.e., either the $c G$ or the $d G$ blow-up time), respectively. Moreover, define

$$
M\left(\varrho, T_{0}\right):=\sup \left\{m: t_{m}(\varrho)=\sum_{l=1}^{m} k_{l}(\varrho) \leq T_{0}\right\}<\infty
$$

and

$$
\Xi\left(u, U, T_{0}\right):=\max \left(\|u\|_{L^{\infty}\left(\left(0, T_{0}\right) ; H\right)},\|U\|_{L^{\infty}\left(\left(0, t_{M\left(\varrho, T_{0}\right)}\right) ; H\right)}\right)<\infty,
$$

where $u$ is the solution of (1.1), and $U$ signifies either the $c G$ or the $d G$ solution. Then, there holds the a priori error estimate

$$
\|u-U\|_{L^{\infty}\left(\left(0, t_{M}\left(\varrho, T_{0}\right)\right) ; H\right)} \leq C\left(T_{0}, \Xi\left(u, U, T_{0}\right)\right) \sqrt{C_{\boldsymbol{r}}} \sqrt{\varrho},
$$

where $C_{\boldsymbol{r}}=\sup _{1 \leq m \leq M\left(\varrho, T_{0}\right)} \max \left(3, \ln \left(r_{m}\right)\right)$, and $C\left(T_{0}, \Xi\left(u, U, T_{0}\right)\right)>0$ only depends on the time $T_{0}$, on $L_{c_{\mathcal{F}}}$, on $\Xi\left(u, U, T_{0}\right)$, and on the constants $c, \alpha, \beta, c_{\mathcal{F}}$, and $\gamma$ from (4.3), (5.1), and (5.3), respectively.

Proof. Let us first suppose that $\Xi\left(u, U, T_{0}\right) \geq c_{\mathcal{F}}$. Then, the operator $\mathcal{F}$ is Lipschitz continuous on the annulus $R_{T_{0}}:=\left\{v \in H: c_{\mathcal{F}} \leq\|v\|_{H} \leq \Xi\left(u, U, T_{0}\right)\right\}$, with Lipschitz constant $L\left(R_{T_{0}}\right)=\gamma \Xi\left(u, U, T_{0}\right)^{\beta-1}$; cf. (5.3). Furthermore, by (5.19) we know that $\mathcal{F}$ is Lipschitz continuous on $\left\{v \in H:\|v\|_{H} \leq c_{\mathcal{F}}\right\}$, with a Lipschitz constant $L_{c_{\mathcal{F}}}$. Moreover, if $\|u\|_{H}<c_{\mathcal{F}}$ and $\Xi\left(u, U, T_{0}\right) \geq\|v\|_{H} \geq c_{\mathcal{F}}$, then we choose $\omega \in[0,1]$ uniquely such that $z_{\omega}:=(1-\omega) u+\omega v$ satisfies $\left\|z_{\omega}\right\|_{H}=c_{\mathcal{F}}$. We deduce that

$$
\begin{aligned}
\|\mathcal{F}(t, u)-\mathcal{F}(t, v)\|_{H} & \leq\left\|\mathcal{F}(t, u)-\mathcal{F}\left(t, z_{\omega}\right)\right\|_{H}+\left\|\mathcal{F}\left(t, z_{\omega}\right)-\mathcal{F}(t, v)\right\|_{H} \\
& \leq L_{\mathcal{F}}\left\|u-z_{\omega}\right\|_{H}+L\left(R_{T_{0}}\right)\left\|z_{\omega}-v\right\|_{H} \\
& \leq\left(\omega L_{\mathcal{F}}+(1-\omega) L\left(R_{T_{0}}\right)\right)\|u-v\|_{H} \\
& \leq \max \left(L_{\mathcal{F}}, L\left(R_{T_{0}}\right)\right)\|u-v\|_{H} .
\end{aligned}
$$

In summary, we conclude that $\mathcal{F}$ is Lipschitz continuous on $\left\{v \in H:\|v\|_{H} \leq\right.$ $\left.\Xi\left(u, U, T_{0}\right)\right\}$, with Lipschitz constant $L_{T_{0}}:=\max \left(L_{\mathcal{F}}, L\left(R_{T_{0}}\right)\right)$. Evidently, due to (5.19), this still holds when $\Xi\left(u, U, T_{0}\right)<c_{\mathcal{F}}$.

Now, we introduce the operator

$$
\mathcal{G}:\left[0, T_{0}\right] \times H \rightarrow H, \quad x \mapsto \begin{cases}\mathcal{F}(t, x) & \|x\|_{H} \leq \Xi\left(u, U, T_{0}\right), \\ \mathcal{F}\left(t, \Xi\left(u, U, T_{0}\right) \frac{x}{\|x\|_{H}}\right) & \|x\|_{H}>\Xi\left(u, U, T_{0}\right),\end{cases}
$$

which is globally Lipschitz continuous on $\left[0, T_{0}\right] \times H$ with Lipschitz constant $L_{T_{0}}$; see Lemma 6. Then, for the cG method, applying [19, Theorem 3.1], we obtain, for $l \in\{0,1\}$, that

$$
\left\|(u-U)^{(l)}\right\|_{L^{2}\left(\left(0, t_{M\left(\varrho, T_{0}\right)}\right) ; H\right)}^{2} \leq C_{\mathrm{cG}}\left(T_{0}, L_{T_{0}}\right)\|u\|_{H^{1}\left(\left(0, T_{0}\right) ; H\right)}^{2} \max _{1 \leq m \leq M\left(\varrho, T_{0}\right)} k_{m}^{2(1-l)},
$$


for a constant $C_{\mathrm{CG}}>0$. Therefore, choosing a time $t^{\star} \in\left[0, t_{M\left(\varrho, T_{0}\right)}\right]$ such that $\|u-U\|_{L^{\infty}\left(\left(0, t_{M\left(o, T_{0}\right)}\right) ; H\right)}=\left\|u\left(t^{\star}\right)-U\left(t^{\star}\right)\right\|_{H}$, and noticing that $U_{0}=u_{0}=u(0)$, we have

$$
\begin{aligned}
\|u-U\|_{L^{\infty}\left(\left(0, t_{M\left(\varrho, T_{0}\right)}\right) ; H\right)}^{2} & =\int_{0}^{t^{\star}} \frac{\mathrm{d}}{\mathrm{d} t}\|u-U\|_{H}^{2} \mathrm{~d} t=2 \int_{0}^{t^{\star}}\left(u-U, u^{\prime}-U^{\prime}\right)_{H} \mathrm{~d} t \\
& \leq 2\|u-U\|_{L^{2}\left(\left(0, t_{M\left(\varrho, T_{0}\right)}\right) ; H\right)}\left\|u^{\prime}-U^{\prime}\right\|_{L^{2}\left(\left(0, t_{M\left(\varrho, T_{0}\right)}\right) ; H\right)} \\
& \leq 2 C_{\mathrm{cG}}\left(T_{0}, L_{T_{0}}\right)\|u\|_{H^{1}\left(\left(0, T_{0}\right) ; H\right)}^{2} \max _{1 \leq m \leq M\left(\varrho, T_{0}\right)} k_{m} \\
& \leq 2 T_{0} C_{\mathrm{cG}}\left(T_{0}, L_{T_{0}}\right)\|u\|_{W^{1, \infty}\left(\left(0, T_{0}\right) ; H\right)}^{2} \max _{1 \leq m \leq M\left(\varrho, T_{0}\right)} k_{m} .
\end{aligned}
$$

Moreover, for the dG time stepping scheme we employ [12, Theorem 3.12] to infer

$$
\|u-U\|_{L^{\infty}\left(\left(0, t_{M\left(\varrho, T_{0}\right)}\right) ; H\right)}^{2} \leq C_{\mathrm{dG}}\left(T_{0}, L_{T_{0}}\right) C_{\boldsymbol{r}}\|u\|_{W^{1, \infty}\left(\left(0, T_{0}\right) ; H\right)}^{2} \max _{1 \leq m \leq M\left(\varrho, T_{0}\right)} k_{m},
$$

where $C_{\mathrm{dG}}>0$ is again a constant. Then, in view of (5.6) and (5.12), we observe that

Thus,

$$
k_{m}(\varrho) \leq \frac{c^{-1} \gamma^{-1} \varrho}{\left\|U_{m-1}\right\|_{H}^{\beta-1}} \leq \frac{c^{-1} \gamma^{-1} \varrho}{\left\|u_{0}\right\|_{H}^{\beta-1}} \leq \frac{c^{-1} \gamma^{-1} \varrho}{c_{\mathcal{F}}^{\beta-1}} .
$$

$$
\begin{aligned}
& \|u-U\|_{L^{\infty}\left(\left(0, t_{M\left(\varrho, T_{0}\right)}\right) ; H\right)}^{2} \\
& \quad \leq \varrho c^{-1} \gamma^{-1} c_{\mathcal{F}}^{1-\beta} \max \left(2 T_{0} C_{\mathrm{cG}}\left(T_{0}, L_{T_{0}}\right), C_{\mathrm{dG}}\left(T_{0}, L_{T_{0}}\right)\right) C_{\boldsymbol{r}}\|u\|_{W^{1, \infty}\left(\left(0, T_{0}\right) ; H\right)}^{2},
\end{aligned}
$$

where $U$ is either the cG or $\mathrm{dG}$ solution. Upon recalling (5.1), we conclude that

$$
\begin{aligned}
\|u\|_{W^{1, \infty}\left(\left(0, T_{0}\right) ; H\right)} & \leq\|u\|_{L^{\infty}\left(\left(0, T_{0}\right) ; H\right)}+\|\mathcal{F}(u)\|_{L^{\infty}\left(\left(0, T_{0}\right) ; H\right)} \\
& \leq \Xi\left(u, U, T_{0}\right)+\alpha \Xi\left(u, U, T_{0}\right)^{\beta},
\end{aligned}
$$

and the proof is complete.

Remark 7. We note that the error estimate (5.20) above is not optimal in terms of $k_{m}$ and $r_{m}$. It is, however, sufficient to establish the blow-up time convergence result in Theorem 3 below.

Lemma 4. Suppose that the assumptions of Proposition 2 hold. Moreover, consider a time $T_{0}>0$ with $T_{\text {sup }}:=\varlimsup_{\varrho} \lim _{\infty} \widetilde{T}_{\infty}(\varrho)>T_{0}$ (note that, by Remark 5, it holds that $\left.T_{\text {sup }}<\infty\right)$. Furthermore, let $\left\{\varrho_{l}\right\}_{l \geq 1}$ be a sequence with $\varrho_{l} \stackrel{l \rightarrow \infty}{\longrightarrow} 0^{+}$that satisfies the bound (5.5), and $\lim _{l \rightarrow \infty} \widetilde{T}_{\infty}\left(\varrho_{l}\right)=T_{\text {sup }}$. Moreover, by Remark 5 , we may suppose that the sequence $\left\{\varrho_{l}\right\}_{l}$ satisfies

$$
\frac{\varrho_{l}\left(\gamma-\alpha \varrho_{l}\right)^{\beta-1}}{c \gamma^{\beta}\left(1-\left(1+C_{0} \varrho_{l}\right)^{1-\beta}\right)} \leq 2 \mu \quad \forall l \geq 1
$$

where $\mu>0$ is the constant from (5.15). Then, whenever $l, m \in \mathbb{N}$ are such that

$$
t_{m}\left(\varrho_{l}\right)=\sum_{i=1}^{m} k_{i}\left(\varrho_{l}\right) \leq T_{0}
$$

with $k_{m}$ from (5.6), the $d G$ and $c G$ time stepping solutions are bounded by

$$
\left\|U_{m}^{-}\left(\varrho_{l}\right)\right\|_{H} \leq\left(\frac{T_{\mathrm{sup}}-T_{0}}{2 \mu}\right)^{1 /(1-\beta)} .
$$

Proof. Suppose that (5.22) holds. Then, applying (5.13) (with $m \rightarrow \infty$ ) it follows that

$$
0<T_{\text {sup }}-T_{0} \leq T_{\text {sup }}-t_{m}\left(\varrho_{l}\right) \leq \frac{\varrho_{l}\left(\gamma-\alpha \varrho_{l}\right)^{\beta-1}}{c \gamma^{\beta}\left(1-\left(1+C_{0} \varrho_{l}\right)^{1-\beta}\right)}\left\|U_{m}^{-}\left(\varrho_{l}\right)\right\|_{H}^{1-\beta}
$$


Using (5.21), we infer that $T_{\text {sup }}-T_{0} \leq 2 \mu\left\|U_{m}^{-}\left(\varrho_{l}\right)\right\|_{H}^{1-\beta}$, which shows the assertion.

Before stating the next lemma, we recall, by Remark 6, that $C_{0} \leq C_{1}$. Hence, for any $A \in \mathbb{R}$ with $A>\left\|u_{0}\right\|_{H}>0$, there holds

$$
A \leq\left(\frac{A}{\left\|u_{0}\right\|_{H}}\right)^{C_{1} / C_{0}}\left\|u_{0}\right\|_{H}<2\left(\frac{A}{\left\|u_{0}\right\|_{H}}\right)^{C_{1} / C_{0}}\left\|u_{0}\right\|_{H} .
$$

Lemma 5. Suppose that the assumptions of Proposition 2 are fulfilled. Furthermore, let $A>\left\|u_{0}\right\|_{H}$. Then, there exists a sequence $\varrho_{l} \stackrel{l \rightarrow \infty}{\longrightarrow} 0^{+}$(satisfying the bound (5.5) ) with

$$
\lim _{l \rightarrow \infty} \widetilde{T}_{\infty}\left(\varrho_{l}\right)=T_{\mathrm{inf}}:=\underline{\lim _{\varrho \searrow 0}} \widetilde{T}_{\infty}(\varrho),
$$

and a time $T_{0}<T_{\inf }$ (depending, in particular, on $A$ and on $\left\|u_{0}\right\|_{H}$ ) such that for any $l$ there is a time index $m_{A}\left(\varrho_{l}\right) \geq 0$ with

$$
t_{m_{A}\left(\varrho_{l}\right)} \leq T_{0}, \quad A \leq\left\|U\left(\varrho_{l}\right)_{m_{A}\left(\varrho_{l}\right)}^{-}\right\|_{H} \leq 2\left(\frac{A}{\left\|u_{0}\right\|_{H}}\right)^{C_{1} / C_{0}}\left\|u_{0}\right\|_{H} .
$$

Here, we denote by $U\left(\varrho_{l}\right)$ either the discrete $c G$ or $d G$ solution from Proposition 2 , and by $\widetilde{T}_{\infty}\left(\varrho_{l}\right)$ the corresponding discrete blow-up time. Furthermore, $C_{0}$ and $C_{1}$ are the constants from (5.11) and (5.17), respectively.

Proof. Due to (5.12), for $\left\|U_{m}^{-}\right\|_{H} \geq A$ to hold, it is sufficient that

$$
m \geq m_{A}(\varrho):=\left\lceil\frac{\ln \left(A\left\|u_{0}\right\|_{H}^{-1}\right)}{\ln \left(1+C_{0} \varrho\right)}\right\rceil .
$$

Then, using (5.18) with $M \geq m_{A}(\varrho)+1 \geq 1$, we have

$$
\begin{aligned}
t_{M}-t_{m_{A}(\varrho)} & \geq \frac{\varrho(\gamma-\alpha \varrho)^{\beta-1}}{c \gamma^{\beta}}\left\|U_{m_{A}(\varrho)}^{-}\right\|_{H}^{1-\beta} \sum_{j=0}^{M-m_{A}(\varrho)-1}\left(1+C_{1} \varrho\right)^{(1-\beta) j} \\
& =\frac{\varrho(\gamma-\alpha \varrho)^{\beta-1}}{c \gamma^{\beta}}\left\|U_{m_{A}(\varrho)}^{-}\right\|_{H}^{1-\beta} \frac{1-\left(1+C_{1} \varrho\right)^{(1-\beta)\left(M-m_{A}(\varrho)\right)}}{1-\left(1+C_{1} \varrho\right)^{1-\beta}} .
\end{aligned}
$$

Letting $M \rightarrow \infty$, leads to

$$
\widetilde{T}_{\infty}(\varrho)-t_{m_{A}(\varrho)} \geq \frac{\varrho(\gamma-\alpha \varrho)^{\beta-1}}{c \gamma^{\beta}} \frac{\left\|U_{m_{A}(\varrho)}^{-}\right\|_{H}^{1-\beta}}{1-\left(1+C_{1} \varrho\right)^{1-\beta}} .
$$

Applying (5.16) $m_{A}(\varrho)$-times, we note that

$$
\left\|U_{m_{A}(\varrho)}^{-}\right\|_{H} \leq\left(1+C_{1} \varrho\right)^{m_{A}(\varrho)}\left\|u_{0}\right\|_{H} \leq\left(1+C_{1} \varrho\right)^{1+\frac{\ln \left(A\left\|u_{0}\right\|_{H}^{-1}\right)}{\ln \left(1+C_{0} \varrho\right)}}\left\|u_{0}\right\|_{H} .
$$

We observe that

$$
\lim _{\varrho \searrow 0}\left(1+C_{1} \varrho\right)^{1+\frac{\ln \left(A\left\|u_{0}\right\|_{H}^{-1}\right)}{\ln \left(1+C_{0} \varrho\right)}}=\left(A\left\|u_{0}\right\|_{H}^{-1}\right)^{C_{1} / C_{0}} .
$$

Furthermore,

$$
\left.\widetilde{T}_{\infty}(\varrho)-t_{m_{A}(\varrho)} \geq \frac{\varrho(\gamma-\alpha \varrho)^{\beta-1}\left\|u_{0}\right\|_{H}^{1-\beta}}{c \gamma^{\beta}\left(1-\left(1+C_{1} \varrho\right)^{1-\beta}\right)}\left(1+C_{1} \varrho\right)^{(1-\beta)\left(1+\frac{\ln \left(A\left\|u_{0}\right\|_{H}^{-1}\right)}{\ln \left(1+C_{0} \varrho\right)}\right.}\right),
$$

and since the right-hand side of the above inequality tends to

$$
\nu:=\frac{\left\|u_{0}\right\|_{H}^{1-\beta}}{c(\beta-1) \gamma C_{1}}\left(A\left\|u_{0}\right\|_{H}^{-1}\right)^{(1-\beta) C_{1} / C_{0}}>0,
$$


as $\varrho \searrow 0$, we conclude that we can choose $\varrho^{\star}$ small enough (and satisfying (5.5) ) so that

$$
\left\|U_{m_{A}(\varrho)}^{-}\right\|_{H} \leq 2\left(A\left\|u_{0}\right\|_{H}^{-1}\right)^{C_{1} / C_{0}}\left\|u_{0}\right\|_{H}, \quad \widetilde{T}_{\infty}(\varrho)-t_{m_{A}(\varrho)} \geq \frac{\nu}{2},
$$

for any $0<\varrho \leq \varrho^{\star}$. Now consider a sequence $\varrho_{l} \stackrel{l \rightarrow \infty}{\longrightarrow} 0^{+}$, with $0<\varrho_{l} \leq \varrho^{\star}$ for all $l$, that satisfies (5.23) as well as

$$
\left|\widetilde{T}_{\infty}\left(\varrho_{l}\right)-T_{\text {inf }}\right| \leq \frac{\nu}{4} \quad \forall l .
$$

Then, upon defining $T_{0}:=T_{\mathrm{inf}}-\nu / 4$, we see that

$$
T_{0}=t_{m_{A}(\varrho l)}+\left(\widetilde{T}_{\infty}\left(\varrho_{l}\right)-t_{m_{A}(\varrho l)}\right)-\left(\widetilde{T}_{\infty}\left(\varrho_{l}\right)-T_{\mathrm{inf}}\right)-\frac{\nu}{4} \geq t_{m_{A}(\varrho l)},
$$

and thus, the proof is complete.

We are now ready to show the following result on the convergence of the Galerkin time stepping schemes to the exact blow-up time.

Theorem 3. Let the assumptions of Proposition 0 and of Lemma 0 be satisfied, and suppose that $\sup _{m \geq 1} r_{m}<\infty$. Then, there holds

$$
\lim _{\varrho \searrow 0} \widetilde{T}_{\infty}(\varrho)=T_{\infty}
$$

where $\widetilde{T}_{\infty}(\varrho)$ denotes either the discrete $c G$ or $d G$ blow-up time, and $T_{\infty}<\infty$ is the blow-up time of (1.1) under the conditions (5.1) and (5.3).

Proof. We establish the proof by contradiction.

Suppose first that $T_{\text {sup }}:=\overline{\lim }_{\varrho \searrow 0} \widetilde{T}_{\infty}(\varrho)>T_{\infty}$. Thence, $\infty>\Delta_{\infty}:=T_{\text {sup }}-$ $T_{\infty}>0$. We can find a sequence $\left\{\varrho_{l}\right\}_{l} \subset \mathbb{R}_{>0}$ satisfying (5.5), with $\varrho_{l} \stackrel{l \rightarrow \infty}{\longrightarrow} 0^{+}$, such that, for all $l$, there holds

$$
\widetilde{T}_{\infty}\left(\varrho_{l}\right) \geq T_{\infty}+\frac{1}{2} \Delta_{\infty}
$$

as well as (5.21). Since the exact solution $u$ of (1.1) blows up at $T_{\infty}$, there is a time $T_{0}, T_{0}<T_{\infty}$, such that

$$
\left\|u\left(T_{0}\right)\right\|_{H} \geq 2\left(\frac{4 \mu}{\Delta_{\infty}}\right)^{1 /(\beta-1)} .
$$

Furthermore, choosing $l^{\prime}$ large enough, there exists a time node index $m\left(\varrho_{l^{\prime}}\right)$ with

$$
T_{0} \leq t_{m\left(\varrho_{l^{\prime}}\right)} \leq \frac{1}{2}\left(T_{0}+T_{\infty}\right),
$$

and such that $\sqrt{\varrho_{l}}$ is sufficiently small. Referring to Lemma 4, we have

$$
\left\|U_{m\left(\varrho_{l^{\prime}}\right)}^{-}\right\|_{H} \leq\left(\frac{T_{\mathrm{sup}}-1 / 2\left(T_{0}+T_{\infty}\right)}{2 \mu}\right)^{1 /(\beta-1)},
$$

uniformly with respect to $\varrho_{l^{\prime}}$. Hence, by virtue of Remark 4 and Lemma 3 (noting that $C_{\boldsymbol{r}}<\infty$ in (5.201) ), it is possible to establish the estimate

$$
\left\|u\left(t_{m\left(\varrho_{l^{\prime}}\right)}\right)-U_{m\left(\varrho_{l^{\prime}}\right)}^{-}\right\|_{H} \leq\left(\frac{4 \mu}{\Delta_{\infty}}\right)^{1 /(\beta-1)} .
$$

Since $t \mapsto\|u(t)\|_{H}$ is non-decreasing this implies that

$$
\begin{aligned}
\left\|U_{m\left(\varrho_{l^{\prime}}\right)}^{-}\right\|_{H} & \geq\left\|u\left(t_{m\left(\varrho_{l^{\prime}}\right)}\right)\right\|_{H}-\left\|u\left(t_{m\left(\varrho_{l^{\prime}}\right)}\right)-U_{m\left(\varrho_{l^{\prime}}\right)}^{-}\right\|_{H} \\
& \geq\left\|u\left(T_{0}\right)\right\|_{H}-\left(\frac{4 \mu}{\Delta_{\infty}}\right)^{1 /(\beta-1)} \geq\left(\frac{4 \mu}{\Delta_{\infty}}\right)^{1 /(\beta-1)} .
\end{aligned}
$$


Then, recalling (5.13), leads to

$$
\begin{aligned}
\widetilde{T}_{\infty}\left(\varrho_{l^{\prime}}\right) & \leq t_{m\left(\varrho_{l^{\prime}}\right)}+\frac{\left\|U_{m\left(\varrho_{l^{\prime}}\right)}^{-}\right\|_{H}^{1-\beta}}{c \gamma^{\beta}} \frac{\varrho_{l^{\prime}}\left(\gamma-\alpha \varrho_{l^{\prime}}\right)^{\beta-1}}{1-\left(1+C_{0} \varrho_{l^{\prime}}\right)^{1-\beta}} \\
& <T_{\infty}+2 \mu\left\|U_{m\left(\varrho_{l^{\prime}}\right)}^{-}\right\|_{H}^{1-\beta} \leq T_{\infty}+\frac{1}{2} \Delta_{\infty},
\end{aligned}
$$

which is a contradiction to (5.24).

Next, let us assume that $T_{\mathrm{inf}}:=\underline{\lim } \varrho \searrow 0_{T_{\infty}} \widetilde{T}_{\varrho}<T_{\infty}$, and define $\Delta_{\infty}:=T_{\infty}-$ $T_{\text {inf }}>0$. Furthermore, let

$$
A:=\max \left(2\left(\delta(\beta-1) \Delta_{\infty}\right)^{1 /(1-\beta)}, 1+\left\|u_{0}\right\|_{H}\right)>\left\|u_{0}\right\|_{H} .
$$

Due to Lemma 5 we can find a sequence $\left\{\varrho_{l}\right\}_{l} \subset \mathbb{R}_{>0}$, with $\varrho_{l} \stackrel{l \rightarrow \infty}{\longrightarrow} 0^{+}$, and a time $T_{0}<T_{\text {inf }}$ so that, for all $l$, there exists $m_{A}\left(\varrho_{l}\right)$ with $t_{m_{A}\left(\varrho_{l}\right)} \leq T_{0}$, and

$$
A \leq\left\|U_{m_{A}\left(\varrho_{l}\right)}^{-}\right\|_{H} \leq 2\left(\frac{A}{\left\|u_{0}\right\|_{H}}\right)^{C_{1} / C_{0}}\left\|u_{0}\right\|_{H}
$$

In particular, $\left\|U_{m_{A}\left(\varrho_{l}\right)}^{-}\right\|_{H}$ is bounded independently of $\varrho_{l}$; evidently, since $T_{0}<$ $T_{\text {inf }}<T_{\infty}$, it follows that $\left\|u\left(t_{m_{A}\left(\varrho_{l}\right)}\right)\right\|_{H}$ is bounded as well. Thus, as before, recalling Remark 4, and using Lemma 3, we may find a sufficiently large index $l^{\prime}$ such that

$$
\left\|u\left(t_{m_{A}\left(\varrho_{l^{\prime}}\right)}\right)-U_{m_{A}\left(\varrho_{l^{\prime}}\right)}^{-}\right\|_{H} \leq \frac{1}{2} A .
$$

Then,

$$
\begin{aligned}
\left\|u\left(t_{m_{A}\left(\varrho_{l^{\prime}}\right)}\right)\right\|_{H} & \geq\left\|U_{m_{A}\left(\varrho_{l^{\prime}}\right)}^{-}\right\|_{H}-\left\|u\left(t_{m_{A}\left(\varrho_{l^{\prime}}\right)}\right)-U_{m_{A}\left(\varrho_{l^{\prime}}\right)}^{-}\right\|_{H} \\
& \geq \frac{1}{2} A \geq\left(\delta(\beta-1) \Delta_{\infty}\right)^{1 /(1-\beta)} .
\end{aligned}
$$

Integrating (5.2) from $t_{m_{A}\left(\varrho_{l^{\prime}}\right)}$ to $T_{\infty}$, we arrive at

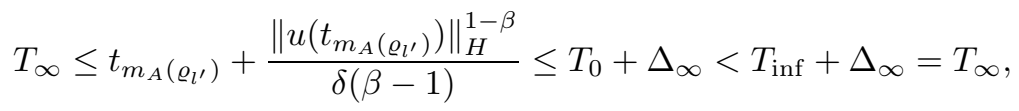

which constitutes a contradiction.

In summary, we have shown that

$$
\varlimsup_{\varrho \searrow 0} \widetilde{T}_{\infty}(\varrho) \leq T_{\infty} \leq \underline{\varrho \searrow 0} \widetilde{T}_{\infty}(\varrho)
$$

which concludes the proof.

5.4. A Time Step Selection Algorithm. The theory in the previous sections suggests the following algorithm for computing a numerical approximation of the exact blow-up time of (1.1) under the conditions (5.1) and (5.3).

Algorithm 1. Suppose that the assumptions of Proposition 2 are satisfied. Choose a parameter $\varrho$ as in (5.5), and a tolerance $\tau>0$. Then:

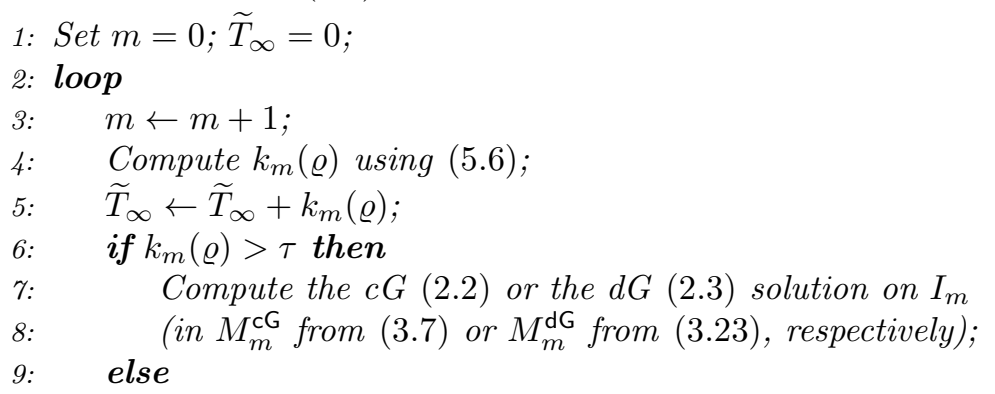




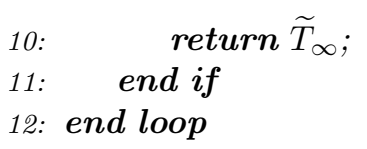

The result, $\widetilde{T}_{\infty}$, is an approximation of the exact blow-up time.

Remark 8. A practical (and platform-independent) implementation of the stopping criterion in the if-statement in line 6 of the above Algorithm 1 is to run the time marching process until

$$
\widetilde{T}_{\infty}+k_{m}(\varrho)==\widetilde{T}_{\infty}
$$

is true, where we make use of the equality operator "==". Note that this also eliminates the need of specifying the tolerance parameter $\tau$.

In order to provide an illustrating example for Algorithm 1, let us consider the initial value problem of finding a function $u=u(t), t \geq 0$, such that

$$
u^{\prime}(t)=\frac{(|u(t)|+1) u(t)}{1+e^{-t}}=: \mathcal{F}(t, u(t)), \quad u(0)=3 .
$$

It has an exact solution $u(t)=3\left(e^{t}+1\right)\left(5-3 e^{t}\right)^{-1}$, and, thus, features a blow-up at $T_{\infty}=\ln (5 / 3)$. Here, $H=\mathbb{R}$, and $c_{\mathcal{F}}<3$ in (5.1) in alignment with Proposition2, Choosing $c_{\mathcal{F}}=2$, a few elementary calculations show that $\alpha=3 / 2, \beta=2$, and $\delta=$ $1 / 2$ in (5.1). Furthermore, $\gamma=5 / 2$ in (5.3), and (5.19) holds with $L_{\mathcal{F}}=5$. The unique positive root of $\Psi$ in (5.4) is given by $\bar{\varrho} \approx 0.243163$. We see that the assumptions of Theorem 3 hold, although, in our computations, we select larger values of $\varrho$ and of $k_{m}(\varrho)$ than would be mandated by (5.5) and (5.6), respectively. More precisely, we consider

$$
k_{m}(\varrho)=\varrho\left|U_{m-1}^{-}\right|^{-1},
$$

for $\varrho \in\left\{2^{-p / 2}, p=4, \ldots, 10\right\}$. We run Algorithm 1 based on the stopping criterion mentioned in Remark 8. The polynomial degree $r=r_{m}$ is kept fixed for all time steps. The results are displayed in Figure 1 (left) for both the cG and the dG time stepping methods for different values of $\varrho$, and for various choices of $r \in$ $\{0, \ldots, 3\}$. The numerical solution on each time step is obtained with the aid of a fixed point iteration as described in Remark 3 (we note that solving the nonlinear problems by means of an adaptive Newton method is potentially more efficient from a computational view point, however, we remark that this approach seems more fragile close to the blow-up due to the large magnitude of the numerical solution). Even though Theorem 3 does not provide any theoretical evidence on convergence rates, the results suggest a convergence to blow-up time of order $\mathcal{O}\left(\varrho^{2(r+1)}\right)$ for the cG method (based on a local polynomial degree $r+1)$, and $\mathcal{O}\left(\varrho^{2 r+1}\right)$ for the $\mathrm{dG}$ scheme (based on a local polynomial degree $r$ ), for $r=0,1,2$; for $r \geq 3$ the errors are too small to allow for a precise identification of the convergence behaviour. The number of time steps was found to be independent of the polynomial degrees (however, strongly dependent on $\varrho$ ) and of whether the $\mathrm{cG}$ or $\mathrm{dG}$ method was employed; see Figure 1 (right).

\section{Conclusions}

In this paper we have investigated the $h p$-version continuous and discontinuous Galerkin time stepping methods for the numerical approximation of general initial value problems with continuous (and possibly unbounded) nonlinearities in real Hilbert spaces. Our main findings include Peano-type existence results for the discrete systems, and a blow-up time step selection algorithm, together with a convergence result, for problems with algebraically growing nonlinearities. We have shown that discrete solutions exist (and are unique within suitable ranges) provided 

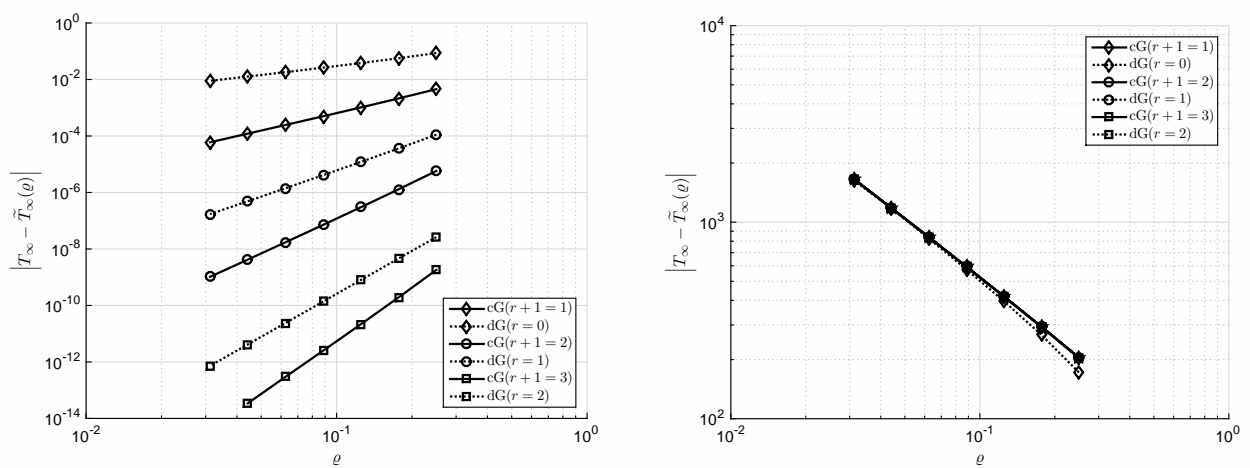

FigURE 1. Errors of approximation of blow-up time for the cG and $\mathrm{dG}$ time stepping schemes for different choices of polynomial degrees (left), and corresponding number of time steps (right).

that the local time steps are chosen sufficiently small (depending on the numerical solutions themselves, however, independent of the local polynomial degrees). The key ingredients in the existence and uniqueness proofs include the derivation of strong forms of the Galerkin discretizations, the transformation into suitable fixed point equations, and the application of fixed point theory. The application of the techniques derived in this article to nonlinear parabolic partial differential equations, and the development of a posteriori error estimates for the blow-up time (in conjunction with the $h p$-framework) are subjects of ongoing research.

\section{Appendix A. An Auxiliary Result}

Lemma 6. Let $\mathcal{F}: H \rightarrow H$ be a continuous function on a (real) Hilbert space $H$, and $M>0$ a constant such that the Lipschitz condition

$$
\|\mathcal{F}(x)-\mathcal{F}(y)\|_{H} \leq L_{M}\|x-y\|_{H}
$$

holds for any $x, y \in H$ with $\|x\|_{H},\|y\|_{H} \leq M$; here $L_{M}>0$ is a constant. Then, the function

$$
\mathcal{G}: H \rightarrow H, \quad x \mapsto \begin{cases}\mathcal{F}(x) & \|x\|_{H} \leq M, \\ \mathcal{F}\left(\frac{M x}{\|x\|_{H}}\right) & \|x\|_{H}>M\end{cases}
$$

is (globally) Lipschitz continuous on $H$ with Lipschitz constant $L_{M}$.

Proof. For $\|x\|_{H},\|y\|_{H} \leq M$ the claim follows immediately from the definition of $\mathcal{G}$ and from (A.1). If $\|x\|_{H},\|y\|_{H}>M$, we have

$$
\|\mathcal{G}(x)-\mathcal{G}(y)\|_{H} \leq L_{M} M\left\|\frac{x}{\|x\|_{H}}-\frac{y}{\|y\|_{H}}\right\|_{H},
$$

where we notice that

$$
\begin{aligned}
\left\|\frac{x}{\|x\|_{H}}-\frac{y}{\|y\|_{H}}\right\|_{H}^{2} & =\frac{2}{\|x\|_{H}\|y\|_{H}}\left(\|x\|_{H}\|y\|_{H}-(x, y)_{H}\right) \\
& \leq \frac{1}{\|x\|_{H}\|y\|_{H}}\left(\|x\|_{H}^{2}+\|y\|_{H}^{2}-2(x, y)_{H}\right)<\frac{1}{M^{2}}\|x-y\|_{H}^{2} .
\end{aligned}
$$

Thus, $\|\mathcal{G}(x)-\mathcal{G}(y)\|_{H}<L_{M}\|x-y\|_{H}$. Moreover, if $\|x\|_{H} \leq M<\|y\|_{H}$, then it holds that

$$
\|\mathcal{G}(x)-\mathcal{G}(y)\|_{H} \leq L_{M} M\left\|\frac{x}{M}-\frac{y}{\|y\|_{H}}\right\|_{H},
$$


where

$$
\begin{aligned}
\left\|\frac{x}{M}-\frac{y}{\|y\|_{H}}\right\|_{H}^{2} & =\frac{1}{M\|y\|_{H}}\|x-y\|_{H}^{2}-\frac{1}{M\|y\|_{H}}\left(\frac{\|y\|_{H}}{M}-1\right)\left(\|y\|_{H} M-\|x\|_{H}^{2}\right) \\
& <\frac{1}{M\|y\|_{H}}\|x-y\|_{H}^{2}<\frac{1}{M^{2}}\|x-y\|_{H}^{2} .
\end{aligned}
$$

Therefore, again $\|\mathcal{G}(x)-\mathcal{G}(y)\|_{H}<L_{M}\|x-y\|_{H}$. The proof for $\|x\|_{H}>M \geq\|y\|_{H}$ follows from symmetry.

\section{REFERENCES}

[1] G. Akrivis, Ch. Makridakis, and R. H. Nochetto. Optimal order a posteriori error estimates for a class of runge-kutta and galerkin methods. Numer. Math., 114:133-160, 2009.

[2] C. Bandle and H. Brunner. Numerical analysis of semilinear parabolic problems with blowup solutions. Real Academia de Ciencias Exactas, Fisicas y Naturales de Madrid. Revista, 88(2-3):203-222, 1994.

[3] W. Bangerth and R. Rannacher. Adaptive Finite Element Methods for Differential Equations. Lectures in Mathematics, ETH Zürich. Birkhäuser Verlag, Basel, 2003.

[4] H. Brunner and D. Schötzau. $h p$-discontinuous Galerkin time-stepping for Volterra integrodifferential equations. SIAM J. Numer. Anal., 44:224-245, 2006.

[5] M. Delfour, W. Hager, and F. Trochu. Discontinuous Galerkin methods for ordinary differential equations. Math. Comp., 36:455-473, 1981.

[6] M. C. Delfour and F. Dubeau. Discontinuous polynomial approximations in the theory of one-step, hybrid and multistep methods for nonlinear ordinary differential equations. Math. Comp., 47(175):169-189, S1-S8, 1986.

[7] D. Estep. A posteriori error bounds, global error control for approximation of ordinary differential equations. SIAM J. Numer. Anal., 32:1-48, 1995.

[8] D. Estep and D. French. Global error control for the continuous Galerkin finite element method for ordinary differential equations. RAIRO Modél. Math. Anal. Numér., 28:815-852, 1994.

[9] M. Frigon and J. W. Lee. Existence principles for Carathéodory differential equations in Banach spaces. Topol. Methods Nonlinear Anal., 1(1):95-111, 1993.

[10] C. Johnson. Error estimates and adaptive time-step control for a class of one-step methods for stiff ordinary differential equations. SIAM J. Numer. Anal., 25:908-926, 1988.

[11] Tomoyasu Nakagawa. Blowing up of a finite difference solution to $u_{t}=u_{x x}+u^{2}$. Applied Mathematics and Optimization. An International Journal with Applications to Stochastics, 2(4):337-350, 1975.

[12] D. Schötzau and C. Schwab. An $h p$ a-priori error analysis of the DG time-stepping method for initial value problems. Calcolo, 37:207-232, 2000.

[13] D. Schötzau and C. Schwab. Time discretization of parabolic problems by the $h p$-version of the discontinuous Galerkin finite element method. SIAM J. Numer. Anal., 38:837-875, 2000.

[14] D. Schötzau and C. Schwab. hp-discontinuous Galerkin time-stepping for parabolic problems. C. R. Acad. Sci. Paris, Série I, 333:1121-1126, 2001.

[15] D. Schötzau and T. P. Wihler. A posteriori error estimation for $h p$-version time-stepping methods for parabolic partial differential equations. Numer. Math., 115(3):475-509, 2010.

[16] A. M. Stuart and M. S. Floater. On the computation of blow-up. European Journal of Applied Mathematics, 1(1):47-71, 1990.

[17] G. Teschl. Ordinary Differential Equations and Dynamical Systems, volume 140. AMS, 9th edition, 2012.

[18] T. Werder, K. Gerdes, D. Schötzau, and C. Schwab. hp-discontinuous Galerkin time-stepping for parabolic problems. Comput. Methods Appl. Mech. Engrg., 190:6685-6708, 2001.

[19] T. P. Wihler. An a-priori error analysis of the $h p$-version of the continuous Galerkin FEM for nonlinear initial value problems. J. Sci. Comput., 25:523-549, 2005. 